\title{
A mathematical model of the effects of hypoxia on the cell-cycle of normal and cancer cells is
}

\author{
T. Alarcón $^{\mathrm{a}, *, 1}$, H.M. Byrne ${ }^{\mathrm{b}}$, P.K. Maini ${ }^{\mathrm{a}}$ \\ ${ }^{a}$ Centre for Mathematical Biology, Mathematical Institute, University of Oxford, 24-29 St Giles', Oxford OX1 3LB, UK \\ ${ }^{\mathrm{b}}$ Division of Applied Mathematics, School of Mathematical Sciences, University of Nottingham, Nottingham NG7 2RD, UK \\ Received 12 November 2003; received in revised form 23 March 2004; accepted 8 April 2004
}

\begin{abstract}
The evolution of the cell-cycle is known to be influenced by environmental conditions, including lack of extracellular oxygen (hypoxia). Notably, hypoxia appears to have different effects on normal and cancer cells. Whereas both experience hypoxia-induced arrest of the $\mathrm{G}_{1}$ phase of the cell-cycle (i.e. delay in the transition through the restriction point), experimental evidence suggests that only cancer cells undergo hypoxia-induced quiescence (i.e. the transition of the cell to a latent state in which most of the cell functions, including proliferation, are suspended).

Here, we extend a model for the cell-cycle due to Tyson and Novak (J. Theor. Biol. 210 (2001) 249) to account for the action of the protein $\mathrm{p} 27$. This protein, whose expression is upregulated under hypoxia, inhibits the activation of the cyclin dependent kinases (CDKs), thus preventing DNA synthesis and delaying the normal progression through the cell-cycle. We use a combination of numerical and analytic techniques to study our model. We show that it reproduces many features of the response to hypoxia of normal and cancer cells, as well as generating experimentally testable predictions. For example our model predicts that cancer cells can undergo quiescence by increasing their levels of p27, whereas for normal cells p27 expression decreases when the cellular growth rate increases.
\end{abstract}

(C) 2004 Elsevier Ltd. All rights reserved.

Keywords: Cell-cycle; Hypoxia; Cancer; p27

\section{Introduction}

The cell-cycle is the set of events whereby a cell duplicates most of its components, including its chromosomes, in order to undergo division (Alberts et al., 1994). The cell-cycle is usually divided into four phases: $G_{1}, S, G_{2}$, and $M$. In $G_{1}(G=$ gap $)$, the cell is not committed to division and the chromosomes do not replicate. Replication of nuclear DNA occurs during the $\mathrm{S}$ phase, whereas completion of mitosis occurs in the final $\mathrm{M}$ phase. The interval between DNA replication

\footnotetext{
This paper was generated using the Academic Press 'generic' LATEXclass file.

*Corresponding author.

E-mail addresses: t.alarcon@cs.ucl.ac.uk, alarcon@maths.ox.ac.uk (T. Alarcón).

${ }^{1}$ Current address: Bioinformatics Unit, Department of Computer Science, University College London, Gower Street, London WC1E 6BT, UK.
}

and division is called the $G_{2}$ phase. The gap phases $G_{1}$ and $G_{2}$ give the cell additional time for growth. The cell also passes through two irreversible transitions. The first of these transitions occurs at the end of $\mathrm{G}_{1}$ and is called "Start" (see Fig. 1 for a schematic representation). During $G_{1}$ the cell monitors its environment and size. When the external conditions and the size of the cell are suitable, the cell commits itself to DNA synthesis and division. This transition is irreversible: once the cell enters the $\mathrm{S}$ phase and DNA replication commences, division has to be completed. The second transition, "Finish", ${ }^{2}$ occurs when DNA replication is completed. Once the cell has checked that DNA and chromatide alignment have occurred, the Finish transition is triggered and the cell finally divides into two daughter cells. A fifth state, the so-called $\mathrm{G}_{0}$ state, is defined to

\footnotetext{
${ }^{2}$ We have adopted the nomenclature used by Tyson and Novak (2001).
} 


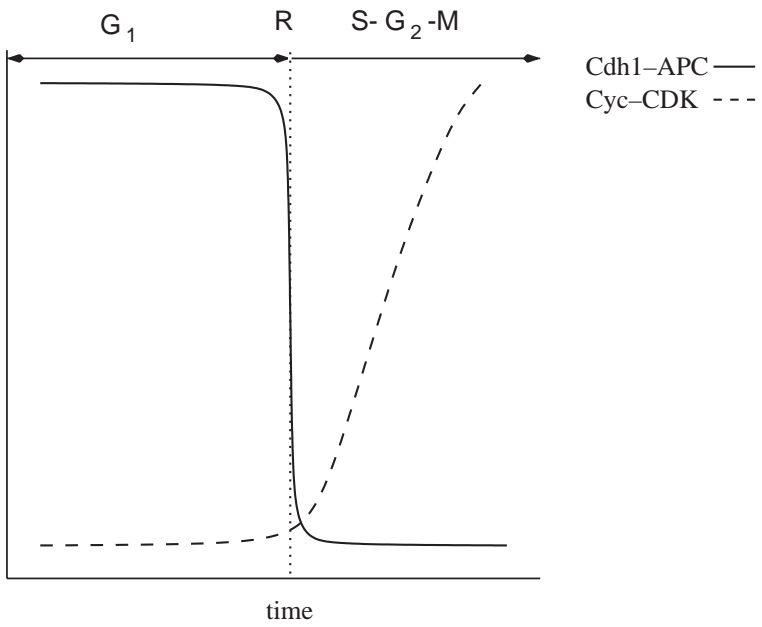

Fig. 1. Schematic diagram showing how intracelluar levels of Cdh1 and Cyc-CDK vary during the cell-cycle. The dotted (vertical) line represents the restriction point $(\mathrm{R})$ at which the "Start" transition occurs. Before the cell goes through this transition, it is in the $\mathrm{G}_{1}$ stage. After the "Start" transition the cell enters the S phase in which DNA synthesis takes place. See text for details.

refer to cells that have abandoned normal progression through the cell-cycle and become quiescent. In this state most (although not all) of the cell functions are suspended, most notably, proliferation

The events occurring during the cell-cycle are controlled by a series of molecular signals. The central components of this network are two families of proteins: the cyclin-dependent kinases (CDKs) and the cyclins (Funk, 1999). The CDKs induce downstream processes by phosphorylating selected proteins. However, to do this, they must be activated by binding to the cyclins. Cyclins are so-called because they undergo a cycle of synthesis and degradation parallel to the cell division cycle. The CDK network is very complex, so that for our modelling purposes we will take into account only its essential features (Tyson and Novak, 2001).

During $\mathrm{G}_{1}$, CDK activity is low because the relevant cyclin partners are missing: their production is inhibited and they are rapidly degraded. At "Start" cyclin synthesis is promoted, and hence the CDKs are activated. CDK activity remains high during $\mathrm{S}, \mathrm{G}_{2}$, and $\mathrm{M}$, since it is necessary for DNA replication and other processes occurring during the final stages of the cycle. At "Finish", the anaphase protein complex (APC) is activated. APC marks specific target proteins (such as cyclins) for degradation by the proteolytic machinery of the cell and is composed of a dozen polypeptides and two auxiliary proteins (Cdc20 and Cdh1). When active, these two proteins present the target proteins to the core of the complex for labelling. Together, they label cyclins for destruction at the end of the cycle, allowing the control system to return to $\mathrm{G}_{1}$. The cyclin-CDK complexes control Cdc20 and Cdh1 activity differently: whilst cyclin-CDK activates Cdc20, it inhibits Cdh1.

The dynamics of the cell-cycle can be affected by environmental conditions, in particular, by the level of extracellular oxygen: it is well documented that low oxygen concentrations (hypoxia) alter progression through the cell division cycle (Gardner et al., 2001), and the $G_{1} / S$ transition, in particular. The response of this transition to hypoxia is mediated by the protein $\mathrm{p} 27$, an element of the CDK network whose production is upregulated under hypoxia (Funk, 1999; Gardner et al., 2001). p27 mediates hypoxia-induced arrest of the $G_{1} / S$ transition by inhibiting Cyc-CDK complex formation and, thereby, inhibiting DNA synthesis.

Hypoxia is a major factor in the evolution of solid tumours. During the avascular phase of tumour growth, cells located in hypoxic regions secrete and release angiogenic factors, thus triggering vascularization of the tumour. In vascularized tumours, hypoxia also plays an important role. Vascular tumours have a heterogeneous composition which may vary over time. For example, cancer cells close to blood vessels proliferate rapidly in the presence of abundant nutrients. Cancer vessels are in general immature (they lack muscular tone) and tend to collapse under the increasing pressure produced by the (unbalanced) tumour cell proliferation. These cells then become hypoxic thereby stimulating a vascular response. In this way, hypoxia contributes to dynamical changes in tumour structure. As we have mentioned above, hypoxia also modulates the rate of progression through the cell-cycle.

The cell-cycles of normal and cancer cells exhibit remarkable differences (Funk, 1999), one of the most important being how they respond to hypoxia: whereas most normal cells undergo apoptosis when the hypoxic stress is too intense or persists for too long, cancer cells appear to have a much higher resistance to hypoxia. This resistance is (in part) due to their ability to enter into a quiescent state under severe or prolonged hypoxic stress (Royds et al., 1998). Of course, if the levels of oxygen become extremely low or hypoxia persists for a long time, the cancer cells eventually die.

Although the role played by $\mathrm{p} 27$ in both normal and cancer cells is the same, there are some differences between the respective patterns of p27 expression. For example the concentration of p27 is, generally, smaller in cancer cells than in normal cells (Bai et al., 2001; Funk, 1999; Philipp-Staheli et al., 2001). It has also been observed that normal cells in the presence of growth factors exhibit reduced levels of p27 expression (Leshem and Halevy, 2002; Saito et al., 2001). In spite of these differences, mutations in the chromosome encoding for p27 are rarely found in human cancer (Funk, 1999). Hence, it is likely that the different behaviour of p27 in normal and cancer cells is produced by different regulatory mechanisms. 
The aim of this paper is to develop a model that reproduces the different responses of normal and cancer cells to hypoxic stress, and, in particular, the ability of cancer cells to enter a quiescent state when oxygen levels fall very low. We also aim to explain why the patterns of p27 expression observed in normal and cancer cells vary.

Related theoretical work on the effects of hypoxia on the behaviour of tumour cells has focused on the role of the tumour suppressor gene p53. Experimental observations indicate that in a hypoxic environment cancer cells carrying a mutant version of the gene p53 have a survival advantage over their wild-type counterparts, This is because mutant cells are less susceptible to hypoxia-induced apoptosis than wild-type cells (Thomson and Royds, 1999). Mathematical models quantifying this survival advantage under repeated rounds of hypoxia have been proposed by Thomson and Royds (1999) and Gammack et al. (2001) for in vitro and in vivo systems, respectively.

As a basic mathematical model for the cell-cycle we use the one developed by Tyson and Novak (2001). A full analysis of this model can be found in Tyson and Novak (2001), so here we only summarize its main features.

Tyson and Novak (2001) claim that regulation of the cell-cycle, i.e. the irreversible transitions "Start" and "Finish", is a consequence of the creation and destruction of stable steady states of the molecular regulatory system of the cell division process. In other words, the core of the cell-cycle is a biological switch (Cherry and Adler, 2000). The set of ordinary differential equations that Tyson and Novak (2001) introduce to model the Start transition is the following:

$\frac{\mathrm{d} x}{\mathrm{~d} t}=\frac{\left(k_{3}^{\prime}+k_{3}^{\prime \prime} A\right)(1-x)}{J_{3}+1-x}-\frac{k_{4} m y x}{J_{4}+x}$,

$\frac{\mathrm{d} y}{\mathrm{~d} t}=k_{1}-\left(k_{2}^{\prime}+k_{2}^{\prime \prime} x\right) y$,

$\frac{\mathrm{d} m}{\mathrm{~d} t}=\mu m\left(1-\frac{m}{m_{*}}\right)$

where $x \equiv[\mathrm{Cdh} 1]$ represents the concentration of active Cdh1/APC complexes, $y \equiv[\mathrm{Cyc}]$, the concentration of cyclin-CDK complexes, ${ }^{3}$ and $m$ is the mass of the cell. The $k_{i}$ 's $(i=1,2,3,4)$ are rate constants and the $J_{i}$ 's $(i=3,4)$ are Michaelis-Menten constants. In Eq. (1), $A$ represents a generic activator. Tyson and Novak (2001) scale their equations so that the total concentration of Cdh1 (active plus inactive) is normalized to 1 and the Michaelis-Menten constants $J_{3}$ and $J_{4}$ are much smaller than 1 . This condition ensures that the model exhibits

\footnotetext{
${ }^{3}$ In Tyson and Novak (2001), [Cyc] corresponds to the concentration of the specific complex cyclinB-CDK. Here we simply consider a generic cyclin-CDK complex in order to keep our model as simple as possible.
}

switching behaviour as in Fig. 1 (Cherry and Adler, 2000). In Eq. (3), $\mu$ is the cell growth rate and $m_{*}$ is the mass of an adult cell.

Eqs. (1)-(3) have been used to model the control that a cell carries out on its size before entering the Start transition (Alberts et al., 1994). This control is incorporated in Eq. (1) through the dependence on the mass of the inactivation term for Cdh1 (see Tyson and Novak (2001) for details).

The model we develop in this paper involves modifying Eqs. (1)-(3) to include the effects of oxygen tension and p27 levels on the cell-cycle. In particular we assume that 227 , whose production is stimulated by hypoxia, will inhibit the formation of Cyc-CDK complexes. Notice that Eqs. (1)-(3) do not constitute a model for the whole cell-cycle; they only account for the $G_{1} / S$ transition. In Tyson and Novak (2001), three additional equations are added to this model in order to account for the latter events that lead to mitosis. However, from Tyson and Novak (2001), we can see that the machinery that finishes the cell-cycle is basically triggered when [Cdh1] is below a threshold value and [Cyc] is above another threshold value. Hence, in order to keep our model as simple as possible, and since the phenomena we are interested in are closely related to the behaviour of $\mathrm{G}_{1} / \mathrm{S}$, we make the simplifying assumption that the cell-cycle is finished when $x=[\mathrm{Cdh} 1]<x_{T H R}$ and $y=$ $[\mathrm{Cyc}]>y_{T H R}$. When this condition is fulfilled the control system is reset (see next section).

The remainder of this paper is structured as follows. In Section 2, we present the experimental results on which our model for normal cells is based (Section 2.1) and formulate the corresponding mathematical model (Section 2.2). We then show how to modify the model to describe cancer cells (Section 2.3). In Section 3, the two models are solved numerically and we verify that our model captures the features observed experimentally. In Section 4, we analyse the phase plane and the fixed points of both models in order to give some insight into the mechanisms responsible for the different qualitative behaviour of normal and cancer cells. Finally, Section 5 contains a summary of our results together with our conclusions.

\section{Model development}

Cell division in both cancer and normal cells is influenced by a range of stimuli. For example, following prolonged periods of overcrowding and/or nutrient deprivation, the cell-cycle is arrested and cells undergo apoptosis (Alberts et al., 1994). One environmental stress that is known to affect cell division is hypoxia (see Carmeliet et al. (1998) and Gardner et al. (2001)). Our aim in this section is to develop simple models for the control of the cell-cycle in normal and cancer cells which 
qualitatively account for experimental observations that indicate their response to hypoxia.

\subsection{Experimental background: normal cells}

In experiments carried out on mouse embryo fibroblasts (MEFs) Gardner et al. (2001) found that hypoxia induces $G_{1}$ arrest and that this is associated with changes in $G_{1}$ regulators. Reduction of retinoblastoma (RB) phosphorylation was observed but no decrease in CDK2 concentration was observed, suggesting that some of the CDKs activity modifiers were affected by hypoxia. They also found that, of all the regulators of CDK2 activity, only cyclinE and p27 were appreciably affected by hypoxia. To establish further the role of $\mathrm{p} 27$, p27 null MEFs were rendered hypoxic. No changes in the cell-cycle profile or in DNA duplication indexes were observed, indicating that p27 may be necessary for hypoxia-induced $\mathrm{G}_{1}$ arrest. As Gardner et al. (2001) remarked, an increase in p27 may be a secondary effect of growth arrest, rather than a mediator of hypoxiainduced $G_{1}$ arrest. However, p27 expression is also increased in null RB MEFs that are cycling under hypoxia, implying that an increase in p27 expression is a consequence of hypoxia, rather than a side effect of RB hypophosphorylation during cell-cycle arrest.

In addition to these observations, there is independent experimental evidence that cyclinA and cyclinE are targets of the E2F transcription factor (DeGregori et al., 1995) and are directly inhibited by hypophosporylated RB (Knudsen et al., 1999). Gardner et al. (2001) summarized these findings by constructing a schematic diagram of hypoxia-induced $\mathrm{G}_{1}$ arrest (see Fig. 3(a)). Here hypoxia leads to overexpression of the CDK inhibitor p27, which downregulates the activity of the cyclin-CDK complexes. This, in turn, prevents the normal progression of the cell through the Start transition.
Throughout this article we will make frequent use of the terms "quiescence" and "arrest". Both terms are used widely in the literature often with slightly different meanings. Therefore to prevent any potential misunderstanding, we now explain how we interpret cell quiescence and arrest. A cell will be said to be in the quiescent state when its progress through the cell-cycle has been halted, i.e. when the cell is in $\mathrm{G}_{0}$. Arrest will refer to a situation in which progress through the cellcycle has been delayed but the cell does not enter $\mathrm{G}_{0}$ (see Fig. 2). Both arrest and quiescence can be induced by hypoxia (low oxygen tension). Our model will allow us to make more precise these definitions. As we show in Section 4 this transition is related to the existence of a bifurcation in the CDK network control system. More specifically, from the dynamical point of view, hypoxiainduced arrest occurs when this bifurcation is delayed whereas hypoxia-induced quiescence occurs when no such bifurcation exists.

It is important to note that in Gardner et al.'s experiments with normal cells no fraction of the population was found in $\mathrm{G}_{0}$, implying that fibroblasts do not appear to exhibit hypoxia-induced quiescence. Hence, we will assume that all normal cells behave similarly, i.e. they exhibit hypoxia-induced arrest (i.e. delay) of the $G_{1} / S$ transition, but not quiescence. If the hypoxic stress is prolonged or becomes too intense, normal cells may undergo apoptosis, usually through the p53 pathway (Kinzler and Vogelstein, 1996). Otherwise, they progress through the different stages of the cell-cycle, however slow this process might be due to arrest of the $G_{1} / S$ transition.

The experimental evidence above suggests that overexpression of p27 (via hypoxia) arrests cell growth. In addition, in the presence of growth factors, expression of p27 in certain cell types is reduced (Leshem and Halevy, 2002; Saito et al., 2001). On the other hand, if growth is arrested for a reason other than hypoxia, p27

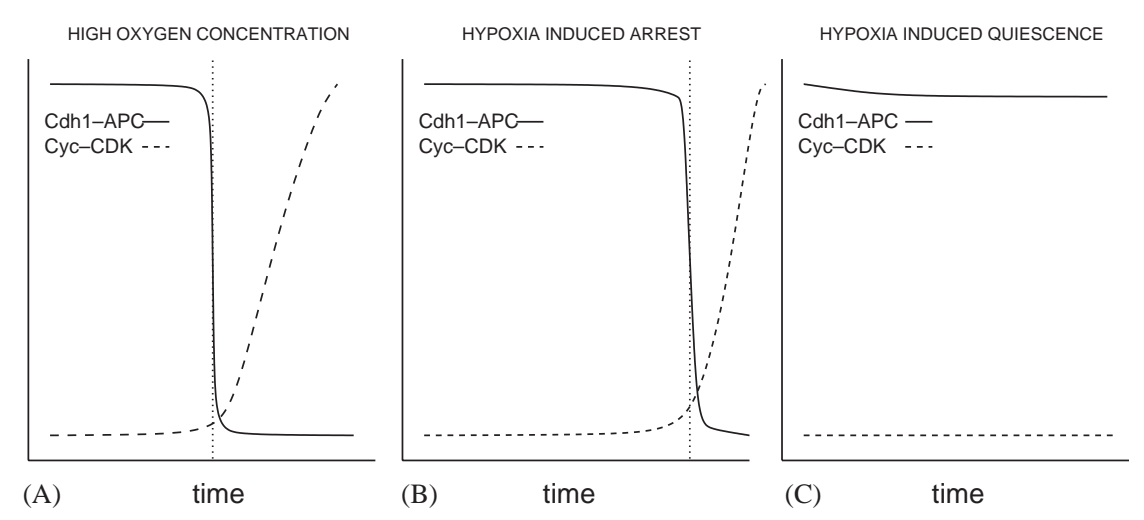

Fig. 2. Schematic diagram showing how intracelluar levels of Cdh1 and Cyc-CDK vary during the cell-cycle in an oxygen-saturated environment (A) and in a hypoxic environment (B and C). Panels B and $\mathrm{C}$ show the difference between hypoxia-induced arrest and quiescence, respectively. Whereas in $B$ the $G_{1} / S$ transition is simply delayed (dotted line), in $C$ such a transition is no longer possible. 


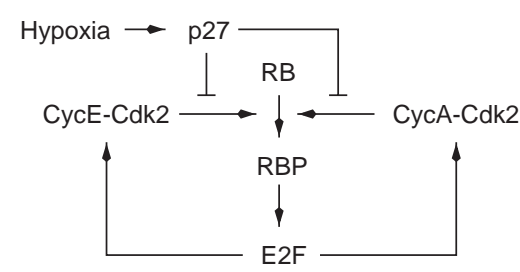

(a)

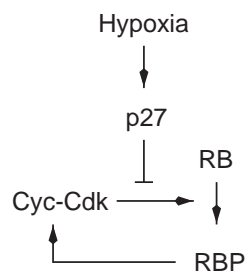

(b)

Fig. 3. Schematic representation of the mechanism for hypoxiainduced cell-cycle arrest in normal cells (fibroblasts). (a) The original mechanism proposed by Gardner et al. (2001). (b) Our simplified version. RBP stands for the phosphorylated form of the RB protein.

is upregulated (Katayama et al., 2000). Thus, expression of p27 is tightly coupled to cell growth.

Our aim now is to incorporate these experimental observations into a mathematical model for hypoxiainduced $\mathrm{G}_{1}$ arrest. We do this by using a simplified version of the mechanism shown in Fig. 3(a): unlike Gardner et al. (2001), we consider only one (generic) cyclin-CDK complex and do not account for E2F explicitly. Instead, we assume that phosphorylated RB (RBP) activates the cyclin-CDK complex directly (Knudsen et al., 1999).

\subsection{Cell-cycle model for normal cells}

Here, we propose a model which reproduces qualitatively the behaviour observed in normal (non-cancerous) cells. In Section 2.3, we modify the model to account for some of the features of cancer cells. The central element of our model of the cell-cycle is the biological switch developed by Tyson and Novak (2001). We modify it to account for the effects of hypoxia and p27 on cell division. The switching behaviour arises from the antagonism between the cyclin-CDK, $y$, and the Cdh1APC complexes, $x$, with the mass of the cell, $m$, triggering the switch. The inhibitory effect of $\mathrm{p} 27, z$, on the cyclin-CDK complexes is incorporated in Eq. (2) through an extra decay term proportional to the concentration of p27 (see Eq. (5)). The equation for the evolution of $z$ (Eq. (7)) derives from the following considerations. Growth affects p27 expression: growth activation downregulates p27 expression whereas growth inhibition increases it. In addition, p27 expression is upregulated by hypoxia.

Another feature we include in our model is the effect of phosphorylated RB. Non-phosphorylated RB (RBNP) is known to inhibit CDK activity (Knudsen et al., 1999) while phosphorylated RB has no direct effect (Gardner et al., 2001). We incorporate this effect into our model by replacing the generic activator $A$ in Eq. (1) by the concentration of RBNP, $u$. Thus, we have indirect activation of CDK through RB phosphorylation. Combining these effects we obtain the following dimensionless model:

$\frac{\mathrm{d} x}{\mathrm{~d} \tau}=\frac{\left(1+b_{3} u\right)(1-x)}{J_{3}+1-x}-\frac{b_{4} m x y}{J_{4}+x}$,

$\frac{\mathrm{d} y}{\mathrm{~d} \tau}=a_{4}-\left(a_{1}+a_{2} x+a_{3} z\right) y$,

$\frac{\mathrm{d} m}{\mathrm{~d} \tau}=\eta m\left(1-\frac{m}{m_{*}}\right)$,

$\frac{\mathrm{d} z}{\mathrm{~d} \tau}=c_{1}\left(1-\frac{m}{m_{*}}\right)-c_{2} \frac{P}{B+P} z$,

$\frac{\mathrm{d} u}{\mathrm{~d} \tau}=d_{1}-\left(d_{2}+d_{1} y\right) u$,

where $P$ is the (non-dimensional) oxygen tension. Cell division is supposed to occur when $x<x_{T H R}$ and $y>$ $y_{T H R}\left(x_{T H R}<y_{T H R}\right){ }^{4}$ The use of this condition is justified by the fact that the biomolecular machinery responsible for completing the cell-cycle is triggered when it is satisfied (Nasmyth, 1995). When this occurs the cell-cycle control is reset by $m \rightarrow m / 2$ and $z \rightarrow 0$. When $m$ and $z$ are so reset the whole control system goes back to the dynamical state corresponding to $G_{1}$, i.e. the corresponding values of $x, y$ and $u$ go back automatically to their " $\mathrm{G}_{1}$ values"; since, as we show in Section 4, when $m=m_{*} / 2$ and $z=0$ the fixed point corresponding to $\mathrm{S}-\mathrm{G}_{2}-\mathrm{M}$ is destroyed and the only stable steady state is $G_{1}$. The instant at which this occurs is defined as the division time, $T_{D}$, assuming that we are starting from $t=0$.

In Eqs. (4)-(8), we have introduced a dimensionless time $\tau=k_{2}^{\prime \prime} t$ with $k_{2}^{\prime \prime}=1 \mathrm{~min}^{-1}$ (Tyson and Novak, 2001). Recall that in the model proposed by Tyson and Novak (2001) the concentrations are dimensionless and all the rate constants have the dimensions of frequency.

Note that our model is slightly different from the model by Tyson and Novak (2001). However, our model is simpler and in the basic facts both models are equivalent. The main difference between the two models is that we are assuming that Eq. (5) is an equation for the active CDK (i.e. the dimers cyc/CDK), whereas Eq. (6) in Tyson and Novak, 2001 corresponds to the total concentration of CDK (active + inactive). Therefore, the last term on the right-hand side of Eq. (5) accounts for the inactivation of CDK. In this way we are able to retain the main characteristics of the control system described in Tyson and Novak (2001) without having to introduce an additional variable to describe, for example, inactive CDK. Both our model and that of Tyson and Novak (2001) exhibit a $\mathrm{G}_{1}$-like steady state (stable when the cell is small) and a $\mathrm{S}_{-} \mathrm{G}_{2}-\mathrm{M}$-like steady state (stable when the cell is full-sized).

\footnotetext{
${ }^{4}$ In the numerical simulations of the normal cell-cycle model Eqs. (4)-(8), we have taken $y_{T H R}=0.2$ and $x_{T H R}=0.004$. For the cancer cell-cycle model, we have taken $y_{T H R}=0.05$ and $x_{T H R}=0.004$.
} 


\subsection{Experimental background and cell-cycle model for cancer cells}

In many respects cancer cells appear to respond differently to hypoxia than their normal counterparts. Although cell-cycle arrest is observed in cancer cells (Funk, 1999), they also have the ability to enter a quiescent state in which most of their functions, including proliferation, are suspended. In a seminal experiment performed by Folkman and Hochberg (1973), an in vitro 3D culture of cancer cells exposed periodically to fresh nutrient solution saturated to a limit size. Furthermore, the resulting multi-cellular spheroid had a layered structure: in the centre of the spheroid, where the nutrient concentration was smallest, there was a core of necrotic material formed by cells that had starved to death. In the outer rim, the nutrient concentration was richest and there was a layer of proliferating cells. A layer of quiescent cells separated these two regions. This experiment suggests that cancer cells, when starved of nutrient, enter a quiescent state before dying. One could argue that this behaviour might not be exclusive to cancer cells. However, we have not found so far direct experimental observation of such a behaviour (i.e. the formation of a quiescent layer) in, for example, multi-cellular spheroids of hepatocytes. Modelling this difference in the behaviour of normal and cancer cells is one of the main aims of this paper.

So, whereas normal cells simply delay their cell division process under hypoxia, cancer cells actually stop it, until the environmental conditions are favourable, by entering into a quiescent state. This is one of the reasons why cancer cells are more resistant to hypoxia than their normal counterparts (Royds et al., 1998). Another difference between cancer and normal cells concerns their rates of expression of p27 (Funk, 1999; Bai et al., 2001; Philipp-Staheli et al., 2001).

We have presented experimental observations supporting the fact that growth regulates (decreases) p27 expression (Leshem and Halevy, 2002; Saito et al., 2001). Our modelling hypothesis is that this control mechanism is absent in cancer cells. In particular whereas the rate at which normal cells produce p27 is assumed to be modulated by their mass, in cancer cells p27 is produced at a constant rate. This is supported by Dhillon and Mudryj (1995) and Park et al. (2001), who report experiments in which the response of p27 in cancer cells to the presence of different growth and proliferation promoting factors is not as expected in normal cells, i.e. the downregulation observed in normal cells is not found in cancer cells. Thus, our mathematical model for the cell-cycle comprises Eqs. (4)-(8), with Eq. (7) replaced by

$\frac{\mathrm{d} z}{\mathrm{~d} \tau}=c_{1}-c_{2} \frac{P}{B+P} z$.
Here, we are assuming that there is a (constant) background production of p27 and simple linear decay modulated by the oxygen tension, $P$.

In the case of the normal cell model we could reset only $m$ and let the value of $z$ readjust by itself. Since, in the cancer model, the p27 dynamics is independent of $m$, we still need to prescribe a resetting mechanism at the end of the cell-cycle. Since we want to analyse the different effects of hypoxia on normal and cancer cells, we introduce as few differences as possible between the two models. This will ensure that we are observing the hypoxia-induced phenomena, rather than "spurious" effects introduced by other factors, and, therefore, we introduce a resetting mechanisms for both models.

\section{Numerical results and comparison to experiments}

We solve our model equations numerically and show that our results are in good qualitative agreement with the experimental observations.

Our model equations were integrated using a standard, four-stage Runge-Kutta method (Press et al., 1992) with initial conditions $x(t=0)=0.9, y(t=0)=$ $0.01, m(t=0)=m_{*} / 2, z(t=0)=0$ and $u(t=0)=1$.

Typical numerical solutions of Eqs. (4)-(8), presented in Fig. 4, show how reducing the oxygen tension extends the cell-cycle by prolonging the duration of $\mathrm{G}_{1}$. On closer scrutiny, our simulations indicate that a reduction in the oxygen tension by a factor $1 / 40-1 / 50$ increases the duration of $\mathrm{G}_{1}$ by $20-25 \%$. This means that in a large population of (identical) cells, we will observe on average approximately $20-25 \%$ more cells in the $\mathrm{G}_{1}$ phase. A similar increase was observed in experiments by Gardner et al. (2001). This can be seen in Fig. 6 where we show how for normal cells the division time, $T_{D}$, varies with the oxygen tension.

The numerical results presented in Figs. 5(a) and (b) show how, for our cancer model (Eqs. (4)-(6) and (8)(9)), the length of the cell-cycle increases as the oxygen tension decreases. However, Fig. 5(c) shows that this increase does not continue indefinitely: for very low oxygen tensions the model predicts that hypoxia causes the cell-cycle to stop. This is consistent with the observed transition of cancer cells to quiescence under hypoxia.

From the mathematical point of view, the system passes through a bifurcation point as $P$ varies: when $P$ exceeds a critical value normal cycling is observed. If, however, $P$ falls below the critical value then the system has only one stable steady state; $G_{1}$ (high concentration of $\mathrm{Cdh}$ and low concentration of active CDK) and cycling is arrested. We can also appreciate this by reference to Fig. 6 which shows how the division time, $T_{D}$, of normal and cancer cells depends on the oxygen tension, $P$. The curve corresponding to the 

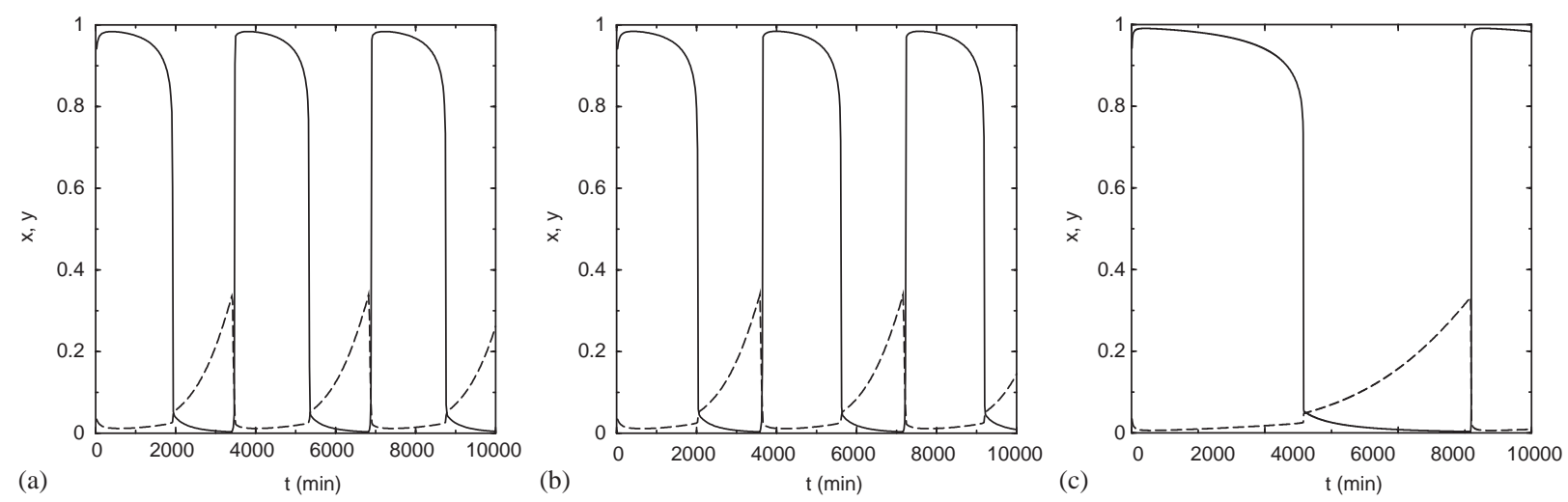

Fig. 4. Series of plots showing how the normal cell-cycle model changes behaviour as the oxygen tension $P$ varies: (a) $P=1$, (b) $P=0.1$, (c) $P=0.001$. Following Tyson and Novak (2001), time is plotted in dimensional units (mins) and the chemical concentrations are plotted in dimensionless units. The parameter values used in these simulations are stated in Table 1. Key: Solid lines correspond to $x$, dashed lines to $y$.
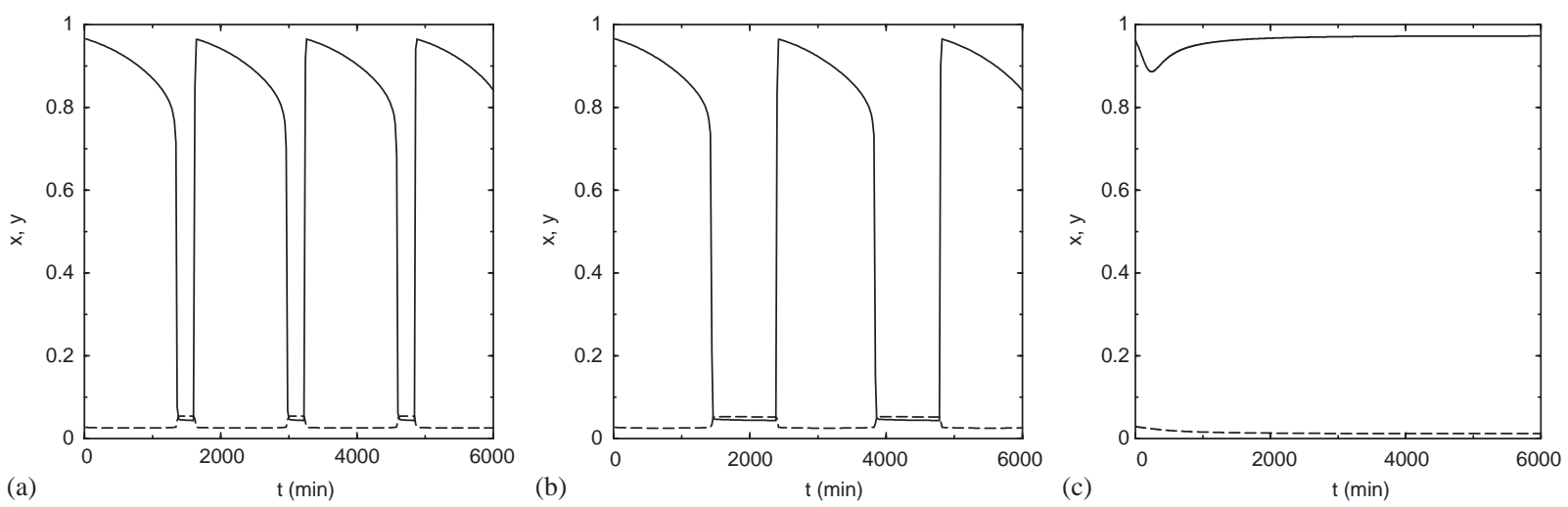

Fig. 5. Numerical solutions of the cancer cell-cycle model for different values of the oxygen tension $P$ : (a) $P=1$, (b) $P=0.1$, (c) $P=0.001$. Solid lines correspond to $x$ and dashed lines to $y$. The parameter values used in these simulations are shown in Table 1 .

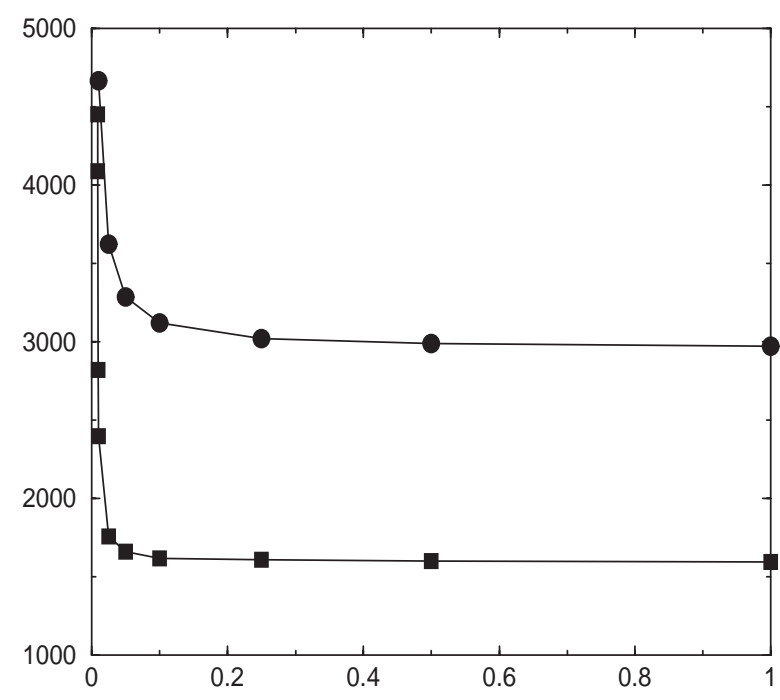

Fig. 6. Division time as a function of the oxygen tension, $P$, for normal cells (circles) and cancer cells (squares). Note the vertical asymptote exhibited by the cancer cells at $P \simeq 0.008$, which is the critical value of the oxygen tension for the transition to quiescence in cancer cells. The parameter values used for these simulations are shown in Table 1. cancer cell-cycle model possesses a vertical asymptote, indicating that $T_{D} \rightarrow \infty$ as $P$ decreases towards a threshold value.

From Fig. 6 we note also that, for the parameter values chosen, the division period of the cancer cells is smaller than that of the normal cells. This is to be expected since the proliferation rate of cancer cells is usually higher than that of their normal counterparts. Our model also reproduces the fact that p27 expression is downregulated for the cancer cells (see Fig. 7). This characteristic feature of cancer cells has been observed by a number of experimental researchers (see for example Funk, 1999; Bai et al., 2001; Philipp-Staheli et al., 2001). Actually, our model predicts that these two features (higher proliferation rate and reduced p27 expression) are closely related.

We now analyse the effects of varying the levels of p27 expression. To this end, we vary $c_{1}$, since this parameter controls the production rate of p27. In Fig. 8 we show how the behaviour of the cancer cell model changes as $c_{1}$ varies. Fig. 8(a) illustrates that the average p27 
concentration,

$$
\langle z\rangle=\frac{1}{T_{D}} \int_{0}^{T_{D}} z(t) d \tau
$$

increases as $c_{1}$ increases. We remark that this is consistent with the increase in the stationary value of $z$ that accompanies an increase in $c_{1}$ (see Eq. (9)). Fig. 8(b) also reveals that as $c_{1}$ decreases (and hence $\langle z\rangle$ decreases) the cells divide more rapidly. In addition the division time $T_{D}$ increases with $c_{1}$, becoming infinite as $c_{1}$ approaches a critical value (which depends on the oxygen tension, $P$ ). Taken together, the results of Figs. 6 and 8 yield a testable model prediction regarding quiescence in cancer cells: quiescence may be induced by either low levels of oxygen or high levels of p27.

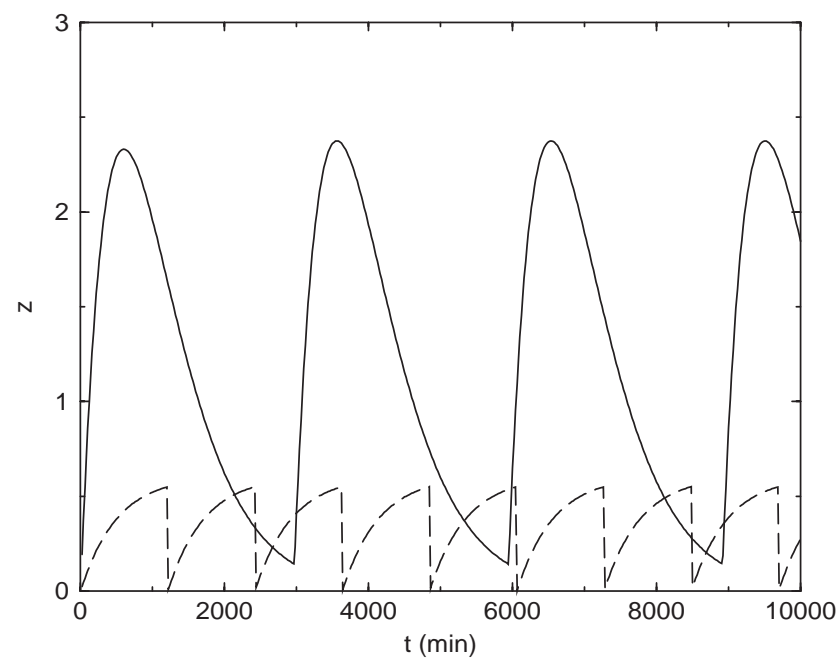

Fig. 7. Diagram showing how levels of p27 expression vary for the normal and cancer cells. The solid line corresponds to normal cells whereas the dashed line corresponds to cancer cells. The parameter values used in these simulations are shown in Table 1 . We have taken $P=1$. See text for details.

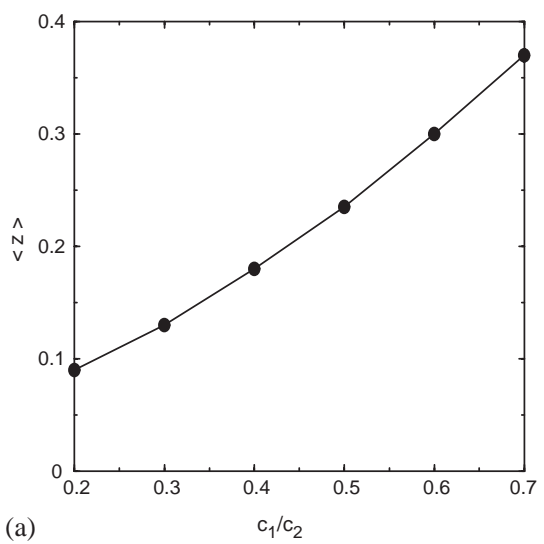

Similar simulations carried out for the normal cell model reveal that varying $c_{1}$ has the same qualitative effect as that described above for the cancer cell model: the concentration of p27 and the division time increase with $c_{1}$. However in this case $T_{D}$ remains finite, which confirms that quiescence cannot be induced in the normal cell-cycle model, at least for the parameter values we are using (results not shown).

This prediction is consistent with a recent clinical study in which low expression of p27 was found to be a poor prognostic indicator in patients with high-grade astrocytomas (Kirla et al., 2003). Within our model framework, we can explain this by observing that low concentrations of p27 are associated with high proliferation rates. In fact, Kirla et al. (2003) argue that expression of p27 in malignant astrocytomas, and its poor prognostic nature, appears to reflect the proliferation activity of tumour cells.

For normal cells p27 levels are affected by the presence of growth factors (Leshem and Halevy, 2002; Saito et al., 2001). Hence, we now investigate the effect of varying the mass growth rate $\eta$ on the behaviour of normal cells. In particular, we solve Eqs. (4)-(8) for different values of the parameter $\eta$ (the mass growth rate) and plot the averaged concentration of p27 as a function of $\eta$. Fig. 9 shows that $\langle z\rangle$ is a decreasing function of the growth rate. Thus, in the presence of a growth factor (i.e. for a bigger growth rate) our model predicts smaller concentrations of $\mathrm{p} 27$. This is consistent with the experimental results of Leshem and Halevy (2002) and Saito et al. (2001), according to which, in the presence of growth factors p27 expression is lower than that in unstimulated control cells.

The results described above support our hypothesis that the two models reproduce the different responses of normal and cancer cells to hypoxia. However, we find one potential problem: Figs. 4 and 5 imply that there is a significant reduction in the concentration of active CDK in the cancer cells. This reduction might be important

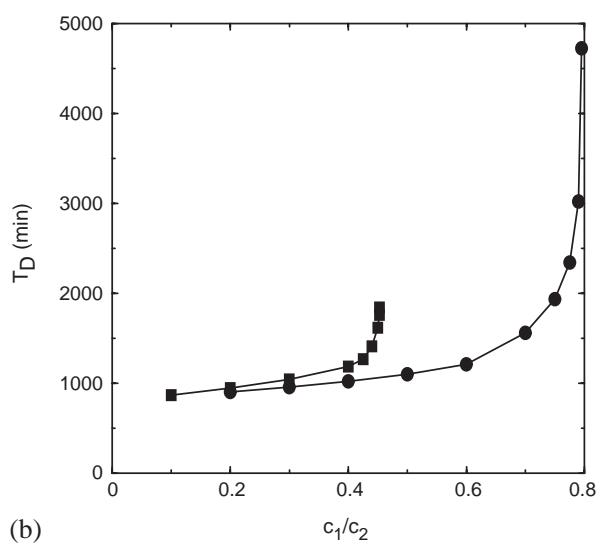

Fig. 8. (a) Averaged concentration of p27, $\langle z\rangle$ (Eq. (10)), in cancer cells as a function of $c_{1} / c_{2}$ for $P=1$. Panel (b) shows the period of division $T_{D}$ of cancer cells as a function of $c_{1} / c_{2}$ for $\mathrm{P}=1$ (circles) and $\mathrm{P}=0.01$ (squares), respectively. The parameter values used for these simulations are shown in Table 1 except for $c_{1}$. We have fixed the value of $c_{2}$ (see Table 1), so the value of $c_{1}$ is given by the corresponding value of $c_{1} / c_{2}$. 


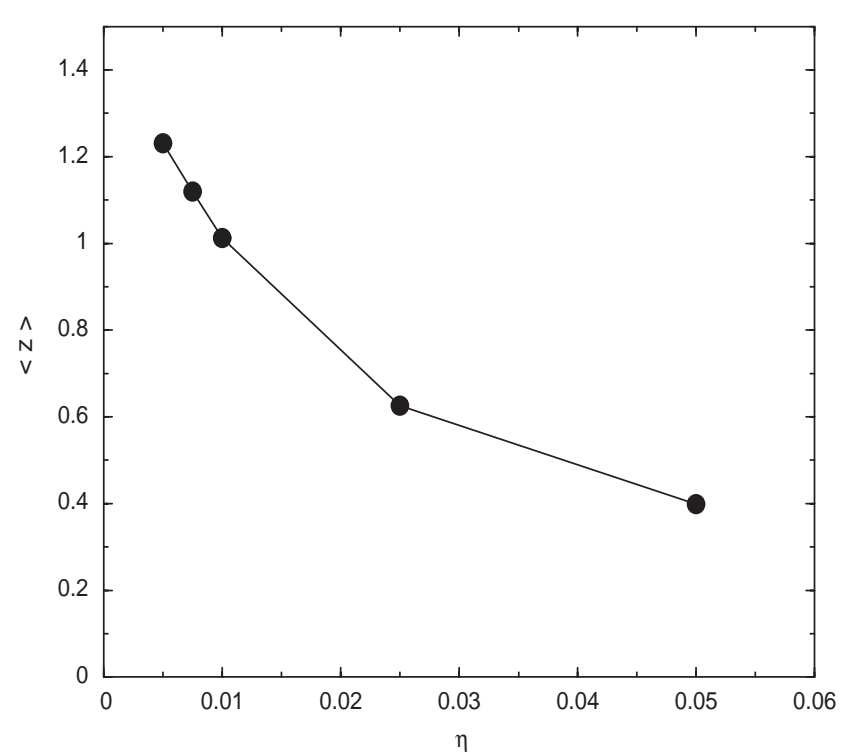

Fig. 9. Averaged concentration of $\mathrm{p} 27,\langle z\rangle$, as a function of the growth rate $\eta$ for normal cells. Consistently (from a qualitative point of view) with the experiments, $\langle z\rangle$ is a decreasing function of $\eta$. In these simulations $P=1$. The rest of the parameter values are taken from Table 1.

since RB-phosphorylation is activated by Cyc-CDK complexes. It could be that the levels of Cyc-CDK predicted by our cancer cell-cycle model are too low to trigger RB-phosphorylation.

\section{Phase plane analysis}

In this section, we use phase plane analysis to explain why, when we use the parameter values given by Tyson and Novak (2001), both models are able to produce arrest but only the cancer model can yield quiescence. In particular, we show that the switching behaviour in these models is controlled by both growth and the kinetics of p27 expression in particular the kinetics used to model p27 expression in normal and cancer cells is responsible for the dramatic differences in their behaviour.

At the beginning of the cell-cycle (at $G_{1}$ ), the cell-cycle control system is in a state in which $x \simeq 1$ and $y \ll x$. Then, according to Tyson and Novak (2001), the system goes through a bifurcation (with the mass of the cell as the control parameter) and changes to a state in which $x \simeq 0$ and $y \gg x$. This bifurcation corresponds to the $\mathrm{G}_{1} / \mathrm{S}$ transition. Hence, for $m=0$ (i.e. at the beginning of the cell-cycle), we expect to find a stationary state with $x \simeq 1$ and $y \ll x$ whereas for $m=m_{*}$ (i.e. towards the end of the cell-cycle), we expect the stationary state should be such that $x \simeq 0$ and $y \gg x$. We will show that, provided we use the parameter values given by Tyson

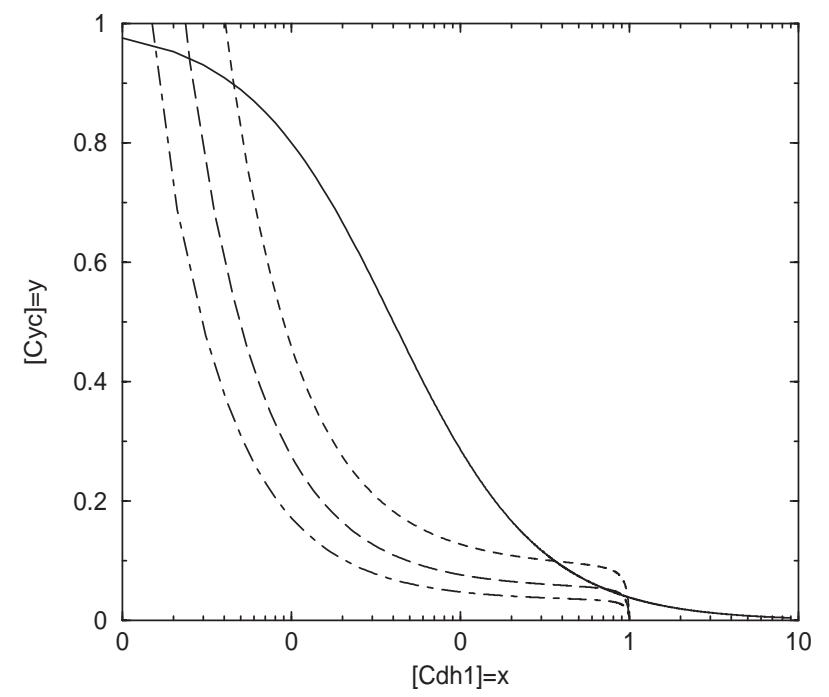

Fig. 10. Phase-plane of Eqs. (1) and (2) for $z_{0}=0, u_{0}=0$. This plot shows the nullclines of these equations for different values of $m$. For $m=0.3$ (dotted line, nullcline of Eq. (1)) this system of equations is bistable, however as $m$ grows and approaches a critical value $m \sim 0.58$ a saddle-node bifurcation occurs. Dashed line corresponds to the nullcline of Eq. (1) with $m=0.5$, dot-dashed line corresponds to the nullcline of Eq. (1) with $m=0.8$. Solid line represents the nullcline of Eq. (2), which does not depend on $m$.

and Novak (2001), the normal cell-cycle model exhibits this behaviour for every value of $P$. On the other hand, the cancer cell-cycle model exhibits this behaviour only if $P$ exceeds a critical value. When $P$ is below this value, the cancer cell-cycle model does not have a steady state with $x \simeq 0$ and $y \gg x$ for $m=m_{*}$. Instead, it gets "stuck" at $x \simeq 1$ and $y \ll x$ (i.e. at $\mathrm{G}_{1}$ ). It is in this sense that we distinguish between arrest and quiescence: whereas for the former the cell will always complete the cell-cycle (probably in such a long time that it would be die via apoptosis first), in the latter case the cell will never complete the cell-cycle since the control system has lost the ability to produce the bifurcation that drives the system through the $G_{1} / S$ transition.

\subsection{Phase portraits}

As in Tyson and Novak (2001), the central control mechanism for our models resides in Eqs. (4) and(5). To start with, let us fix $z=z_{0}, m=m_{0}, u=u_{0}$ in Eqs. (4) and (5), where $z_{0}, m_{0}, u_{0}$ are arbitrary. When $z_{0}=0$ and $u_{0}=0$, Tyson and Novak (2001) observed that Eqs. (4) and (5) exhibit a saddle-node bifurcation when $m_{0}$ takes a critical value ${ }^{5}$ (see Fig. 10). For smaller values of $m_{0}$ Eqs. (4) and (5) have two stable fixed points (SFP) and an unstable fixed point (UFP). As mentioned in the

\footnotetext{
${ }^{5}$ Tyson and Novak (2001) estimate this critical value to be about 0.58 for the parameter values stated in Table 1 .
} 

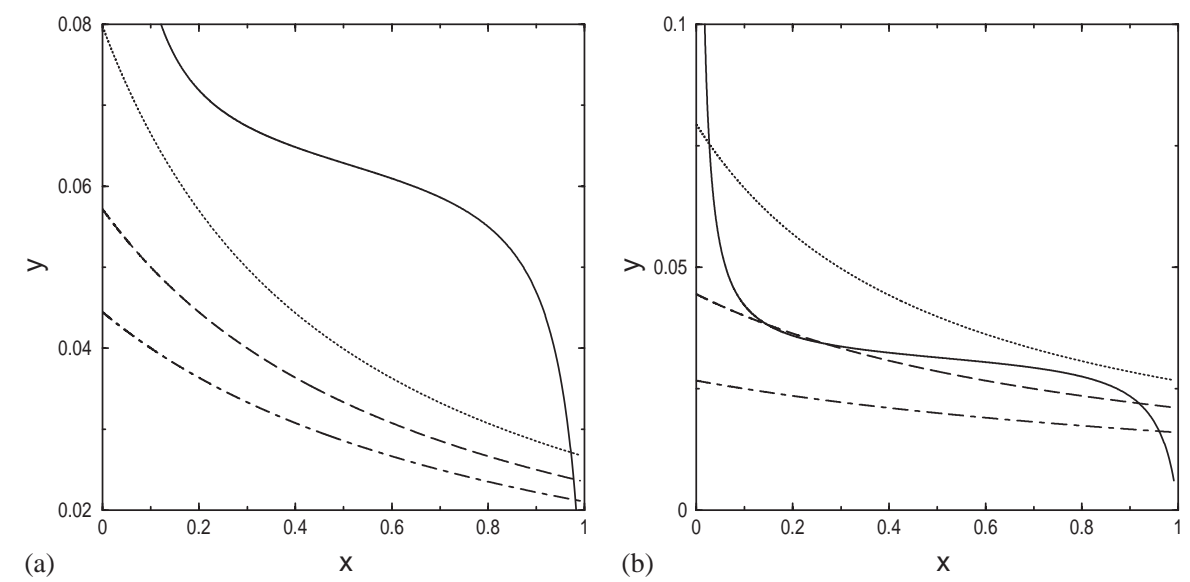

Fig. 11. Phase-plane of Eqs. (4) and (5) showing their steady states for different values of $z$ and $m$. (a) Nullcline of Eq. (7) for different values of $z$ : dotted line corresponds to $z=0.81$, dashed line to $z=1.6$, dot-dashed line to $z=2.4$. Solid line corresponds to the nullcline of Eqs. (5) with $m=5$. (b) Nullclines of Eq. (5) for different values of $z$ : dotted line corresponds to $z=0.81$, dashed line to $z=2.4$, dot-dashed line to $z=4.8$. Solid line corresponds to the nullcline of Eq. (4) with $m=10$.

previous section, one SFP represents $G_{1}$ and the other is $\mathrm{S}-\mathrm{G}_{2}-\mathrm{M}$. When $m_{0}$ increases towards its critical value $\mathrm{G}_{1}$ collides with the UFP and a saddle-node bifurcation occurs. Only the $\mathrm{S}_{-} \mathrm{G}_{2}-\mathrm{M}$ fixed point persists for larger values of $m_{0}$.

Let us see how this picture is modified as $z$ varies. The corresponding phase-plane, presented in Fig. 11, shows that when $m_{0}=5$ (Fig. 11(a)) Eqs. (4) and (5) have only one steady state, regardless of the value of $z_{0}$.

The picture is qualitatively different when $m_{0}$ has a higher value, say $m_{0}=m_{*}$. In this case the variation of $z_{0}$ produces a switching behaviour: for values of $z_{0}$ smaller than some critical value the only SFP is the $\mathrm{S}-\mathrm{G}_{2}-\mathrm{M}$ fixed point, whereas for $z_{0}$ larger than its critical value the only SFP is the $G_{1}$ fixed point.

Thus, for the normal cells, the situation is as follows. Referring to Fig. 7 we recall that over time the concentration of $\mathrm{p} 27$ grows, reaches a maximum value and then decreases. This pattern is repeated cyclically each time the cell divides. At the end of the cycle p27 is decreasing and therefore, according to Fig. 11(b), the system is driven towards the $\mathrm{S}-\mathrm{G}_{2}-\mathrm{M}$ fixed point.

The behaviour of cancer cells can also be explained in terms of the phase-plane shown in Fig. 11. According to Fig. 7, the concentration of p27 grows monotonically to a saturating (steady state) value, until the control system is reset to its initial condition. This steady state value, $z_{\infty}=c_{1}(B+P) / c_{2} P$, depends on the oxygen concentration, $P$. When $P$ is sufficiently small $z_{\infty}$ exceeds a critical value and, as shown in Fig. 11(b), the control system gets trapped at the $G_{1}$ fixed point. This brings the cellcycle to a halt. If, on the contrary, the oxygen concentration is such that $z_{*}$ is smaller than the critical value, the system oscillates by the same mechanism as for the normal cells.
A more rigorous analysis of all these features is presented in Appendix A.

\subsection{Summary of results of the phase plane analysis}

The results of Appendix A are summarized here and illustrated by Fig. 12. This figure shows the bifurcation diagrams of the normal (Figs. 12(a) and (b)) and the cancer (Figs. 12(c) and (d)) models, excluding the equation for the mass, which is viewed as a control parameter. To obtain these diagrams we have computed numerically the steady state values $x_{\infty}, y_{\infty}, z_{\infty}$, and $u_{\infty}$ for different values of the cell mass and the oxygen level. Stability of the steady state solutions is determined by linearizing the model equations. Figs. 12(a) and (c) show the bifurcation diagrams for normal and cancer cells, respectively, when $m$ is the control parameter and the oxygen level is $P=1$. The figures show that the switching behaviour of each system is driven by growth: as $m$ increases the systems go through successive bifurcations which produce the transition from $G_{1}$, the stable fixed point for small values of $m$, to $\mathrm{S}-\mathrm{G}_{2}-\mathrm{M}$, the stable fixed point when $m$ approaches its saturation value. This agrees with the results obtained from our linear stability analysis: for large values of the oxygen level (as $P=1$ ), these are the stable fixed points for the corresponding values of $m$.

Differences between the two models appear when we consider low oxygen levels. Our linear stability analysis has revealed that $G_{1}$ is the only fixed point at the beginning of the cell-cycle (i.e. small values of $m$ ) for both models. So, we focus on the end of the cell-cycle when $m=m_{*}$ and take $P$ as the control parameter. Figs. 12(b) and (d) show the dramatic difference between the models as $P$ varies. While the behaviour 

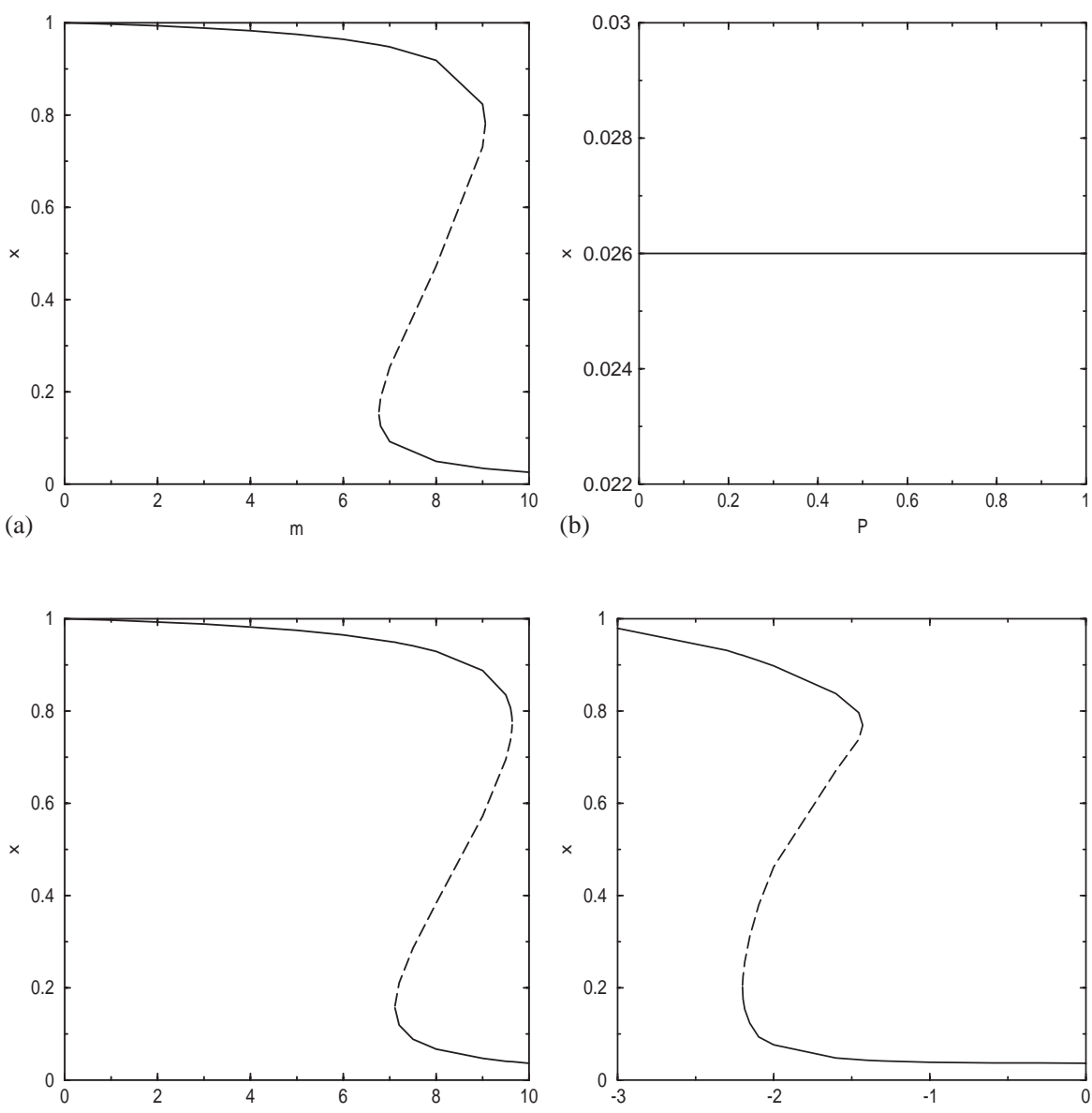

(c)

$\mathrm{m}$

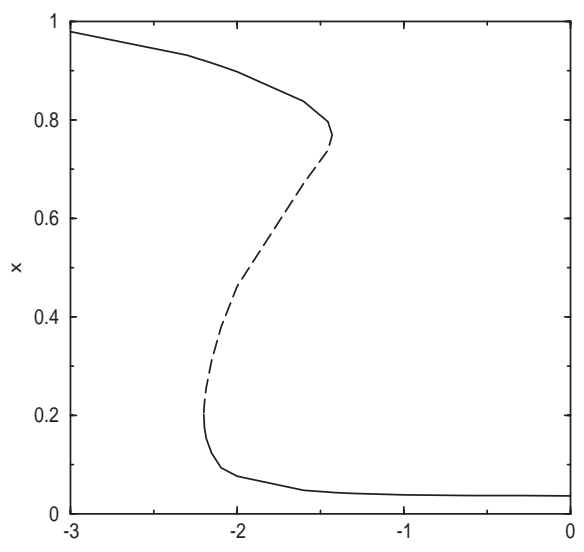

(d)

$\log (\mathrm{P})$

Fig. 12. Bifurcation diagrams for our two models. Panels (a) and (b) correspond to the normal cell-cycle model. In (a) we have fixed $P=1$ and taken $m$ as the control parameter, whereas in (b) $P$ plays the role of the control parameter with $m=m_{*}$. Panels (c) and (d) correspond to the cancer cellcycle model. In (d) we have fixed $P=1$ and taken $m$ as the control parameter. In (c) $P$ plays the role of the control parameter with $m=m_{*}$. Comparing panels (a) and (c) we can observe that the critical value of the cell mass in the cancer model is bigger than in the normal model.

of the normal cell model (Fig. 12(b)) is independent of $P$ $\left(\mathrm{S}-\mathrm{G}_{2}-\mathrm{M}\right.$ is the stable fixed point for all values of $P$ ), the stable fixed point of the cancer model depends on $P$ : when $P$ is smaller than some critical value the stable fixed point is $\mathrm{G}_{1}$ (thus yielding quiescence) whereas for $P$ larger than the critical value the stable fixed point is $\mathrm{S}_{-} \mathrm{G}_{2}-\mathrm{M}$. This is consistent with our linear stability analysis and the phase-portrait-based discussion of Section 3.1, where we saw that quiescence in the cancer cell model is related to high values of $z$ towards the end of the cell-cycle for this model, high values of $z$ occur when the oxygen tension is low.

Throughout this paper, we have emphasized that normal cells do not have the ability to enter a quiescent state under hypoxia. However, in Section A.1 we have shown that our normal cell model can produce quiescence if $b_{4} m_{*} \simeq O(1)$. This allows for the possibility of having quiescence in normal cells due to mechanisms (different from hypoxia) that drive the system into this parameter regime.

\section{Discussion}

In this paper, we have adapted an existing mathematical model of the cell-cycle to describe the behaviour of normal and cancer cells, focussing on the differences in their responses to hypoxia. Our models are successful in producing hypoxia-induced arrest of the $\mathrm{G}_{1} / \mathrm{S}$ transition, in agreement with experimental observations (Funk, 1999; Gardner et al., 2001). In the case of normal cells, the relative increase in the duration of the cell-cycle is similar to that observed by Gardner et al. (2001). However, whilst both models can produce arrest, only the cancer cell-cycle model is able to yield quiescence, as long as we use the parameter values given in Table 1, which are due to Tyson and Novak (2001). However, in other parameter regimes, the normal cell-cycle model might produce quiescence.

The difference between the two models stems from differences in their rates of $\mathrm{p} 27$ expression: for normal cells p27 expression is growth-regulated (i.e. it depends 
Table 1

Summary of those (non-dimensional) parameter values that are held fixed throughout the paper, unless otherwise stated

\begin{tabular}{lccl}
\hline Parameter & $\begin{array}{l}\text { Value } \\
\text { (normal) }\end{array}$ & $\begin{array}{l}\text { Value } \\
\text { (cancer) }\end{array}$ & Source \\
\hline$a_{4}$ & 0.04 & 0.04 & Tyson and Novak (2001) \\
$a_{1}$ & 0.5 & 0.4 & Tyson and Novak (2001) \\
$a_{2}$ & 1 & 1 & Tyson and Novak (2001) \\
$a_{3}$ & 0.25 & 0.25 & \\
$b_{3}$ & 10 & 10 & Tyson and Novak (2001) \\
$b_{4}$ & 35 & 35 & Tyson and Novak (2001) \\
$\eta$ & 0.01 & 0.01 & Tyson and Novak (2001) \\
$m_{*}$ & 10 & 10 & Tyson and Novak (2001) \\
$J_{3}, J_{4}$ & 0.04 & 0.04 & Tyson and Novak (2001) \\
$c_{1}$ & 0.1 & 0.007 & \\
$c_{2}$ & 0.01 & 0.01 & \\
$B$ & 0.01 & 0.01 & \\
$d_{1}$ & 0.01 & 0.01 & \\
$d_{2}$ & 0.1 & 0.1 & \\
\hline
\end{tabular}

The values of the parameters for which we have not found estimates in the literature have been calculated to fit the values of the duplication times of V-79 cancer cells reported by Deutsch and Dormann (2002). According to these authors the division time for V-79 cells is between 13 and $19 \mathrm{~h}$ (780 and $1140 \mathrm{~min}$, respectively), which is in good agreement with our predicted division time for cancer cells exposed to high concentrations of oxygen (see Fig. 6).

on the cell mass) whereas for the cancer cells it is growth-independent. We justify these modelling assumptions by appealing to experiments by Leshem and Halevy (2002) and Saito et al. (2001). They found that in the presence of growth factors normal cells reduced their rates of p27 expression whereas cancer cells did not (Dhillon and Mudryj, 1995; Park et al., 2001). Another fact supporting our hypothesis is that mutations of the p27 gene are rarely found in human tumours (Funk, 1999), supporting the fact that changes in the mechanism regulating its expression might play a key role in alterating the behaviour of cancer cells.

Our model also reproduces other experimental observations: higher proliferation rates in cancer than in normal cells (see Fig. 6 and Funk, 1999), lower levels of the protein p27 in cancer than in normal cells (see Fig. 7 and Funk, 1999; Bai et al., 2001; Philipp-Staheli et al., 2001) and, in the case of the normal cell model, lower levels of p27 in the presence of growth factors (i.e. higher values of $\eta$ ) (see Fig. 9 and Leshem and Halevy, 2002; Saito et al., 2001). Our results are also consistent with a clinical study which showed that low levels of p27 were a poor prognostic factor, since low levels of p 27 are related to an increase in the proliferation rate (see Fig. 8 and Kirla et al., 2003). In addition our model predicts that quiescence in cancer cells could be induced by increasing p27 levels. This is experimentally testable.

While our model is consistent with a number of experimental observations and generates experimentally testable predictions, it possesses some shortcomings that require further refinement. For example, it does not deal properly with the end of the cell-cycle: in our simulations the division cycle finishes when $x<x_{T H R}$ and $y>$ $y_{T H R}\left(y_{T H R}<x_{T H R}\right)$, where $x=[\mathrm{Cdh}]$ and $y=[\mathrm{Cyc}]$. Biologically, the justification for this is that under this condition the biomolecular machinery responsible for completing the cell-cycle is triggered. A more accurate description of the "Finish" transition, as in Tyson and Novak (2001), is postponed for future work.

Our model should also incorporate hypoxia-induced apoptosis, since both normal and cancer cells undergo programmed cell death when the level of oxygen is very low, with even the cancer cells dying when exposed to a prolonged hypoxic stress.

It is important to stress that whilst we have focused on the role of the protein p27 as a mediator of cell response to oxygen starvation, in particular hypoxia-induced arrest and quiescence, other mechanisms involving different pathways may be relevant. We have shown in our linear stability analysis that the normal cell-cycle model cannot produce quiescence, since our parameter values are such that $b_{4} m_{*} \simeq O\left(\varepsilon^{-1}\right)$. However, the normal cell-cycle model would exhibit quiescence if $b_{4} m_{*} \simeq O(1)$, where $m_{*}$ is the maximum size of a fully grown cell and $b_{4}$ accounts for the intensity of the coupling between growth and $[\mathrm{Cdh}]$ dynamics. Thus, possible mechanisms to induce quiescence in this model would be to reduce the value of either $b_{4}$ or $m_{*}$. Whether this is biologically feasible should be the subject of further experimental investigation.

We have assumed that normal cells do not enter quiescence under oxygen starvation, although quiescence induced by other mechanisms is not excluded. On the contrary, in experiments carried out in tumour multicellular spheroids starvation induced quiescence has been observed. On the other hand, Hamamoto et al. (1998) reported that hepatocytes cultured as spheroids grew more slowly than hepatocytes cultured in 2D monolayer cultures. ${ }^{6}$ Also a higher concentration of p27 was observed in the spheroids. Both of these facts might be signatures of quiescence. However, to the best of our knowledge, no direct observation of a quiescent region in normal cell spheroids has been reported so far. On the other hand, our normal cell-cycle model can explain most of the behaviour observed in the hepatocyte experiments: the increased p27 levels would be caused by the reduction in oxygen and this, in turn, would diminish the proliferation rate (see Section 3). The smaller growth rate could be caused by a balance

\footnotetext{
${ }^{6}$ In these experiments, nutrient is available to all the cells in the monolayer culture. However, in 3D spheroids nutrient availability is limited, since it enters the spheroid by diffusion through the surface of the spheroid. This fact explains why on average spheroids experience lower oxygen tensions than monolayer cultures.
} 
between proliferation and death, rather than being produced by a quiescent population of cells.

There is one factor that has not been taken into account in this article, but that might be important, that is the relationship between growth and progression through the cell-cycle. The average size (diameter) of a normal cell is estimated to be approximately $10 \mu \mathrm{m}$, although the variance associated with this figure is very large. Experiments performed by Sauer et al. (1998) report measurements of the size of quiescent cancer cells in multicellular spheroids averaging $13 \mu \mathrm{m}$ in diameter. They also find that cycling cancer cells within the spheroids grow to $18 \mu \mathrm{m}$ in diameter. This reveals an intricate relationship between growth and cell-cycle, which is neglected in our model where growth is considered as an autonomous process.

To summarize, we have proposed models for the hypoxia-induced response of the $\mathrm{G}_{1} / \mathrm{S}$ transition in both normal and cancer cells. Our models reproduce a range of experimental observations on each type of cell. In particular, our simulations provide a mechanism to understand how cancer cells may become quiescent under low levels of oxygen. We have been able to define this property in mathematical terms: whereas the $G_{1} / S$ transition in normal and nonquiescent cancer cells is driven by a bifurcation in the dynamics of the CDK network, quiescent cells are characterized by the absence of such a bifurcation.

\section{Acknowledgements}

TA thanks the EU Research Training Network (5th Framework): "Using mathematical modelling and computer simulation to improve cancer therapy" for funding this research. HMB thanks the EPSRC for funding as an Advanced Research Fellow. This research (PKM) was supported in part by the National Science Foundation under Grant No. PHY99-07949. PKM would like to thank KITP, UC Santa Barbara for their kind hospitality.

\section{Appendix A. Fixed points and linear stability analysis of the normal and cancer models}

Here we give the details of the analysis corresponding to the discussion presented in Section 4.

\section{A.1. Fixed points and linear stability analysis of the normal cell model}

Before proceeding further, we simplify Eqs. (4)-(8). From the numerical solutions, we note that throughout most of the cell-cycle either $x \simeq 0$ (in $\mathrm{S}_{-} \mathrm{G}_{2}-\mathrm{M}$ ) or $x \simeq 1$ (in $\mathrm{G}_{1}$ ). To start with, we assume $x=1-\varepsilon \tilde{x} \gg J_{4}$ where $0<\varepsilon \ll 1^{7}$ and we obtain

$\varepsilon \frac{\mathrm{d} \tilde{x}}{\mathrm{~d} \tau}=b_{4} m y-\varepsilon\left(1+b_{3} u\right) \tilde{x}$,

$\frac{\mathrm{d} y}{\mathrm{~d} \tau}=a_{4}-\left(a_{1}+a_{2}+a_{3} z\right) y$,

$\frac{\mathrm{d} m}{\mathrm{~d} \tau}=\eta m\left(1-\frac{m}{m_{*}}\right)$,

$\frac{\mathrm{d} z}{\mathrm{~d} \tau}=c_{1}\left(1-\frac{m}{m_{*}}\right)-c_{2} \frac{P}{B+P} z$,

$\frac{\mathrm{d} u}{\mathrm{~d} \tau}=d_{2}(1-u)-d_{1} y u$.

Eq. (A.3) is decoupled and has two fixed points: $m_{\infty}=0$ and $m_{\infty}=m_{*}$. The case $m_{\infty}=m_{*}$ is incompatible with Eq. (A.1), unless $b_{4} m_{*} y_{\infty}=O(\varepsilon)$. However, this is not the case if we use the parameter values recorded in Table 1, for, although $y_{\infty}=O(\varepsilon), b_{4} m_{*}=$ $350=O\left(\varepsilon^{-1}\right)$. If $b_{4} m_{*}=O(1)$ we would have a $\mathrm{G}_{1}$ type fixed point with $m_{\infty}=m_{*}$ and, therefore, that would yield quiescence in the normal cell-cycle model. This may raise the question of how robust our results are to changes in the parameters given by Tyson and Novak (2001). We have made additional analysis and run numerical simulations to check robustness with respect to $b_{4} m_{*}$ (results not shown). We have found that our results are robust to changes in this parameter, although when $b_{4} m_{*}=O(1)$ the normal cell-cycle model is seen to exhibit quiescent behaviour.

However, if we use the parameter values from Table 1, only $m_{\infty}=0$ is feasible and we obtain, at leading order:

$z_{\infty}=\frac{c_{1}(B+P)}{c_{2} P}$,

$y_{\infty}=\frac{a_{4}}{a_{1}+a_{2}+a_{3} z_{\infty}}$,

$u_{\infty}=\frac{d_{2}}{d_{2}+d_{1} y_{\infty}}$,

with $x_{\infty}=1$.

The stability of this fixed point can be analysed by linearizing Eqs. (A.2), (A.4), and (A.5). Writing $\delta y=$ $y-y_{\infty}, \delta z=z-z_{\infty}$, and $\delta u=u-u_{\infty}$ in Eqs. (A.2),

\footnotetext{
${ }^{7}$ In this section $\varepsilon=0.04$ is of the same order of magnitude as $a_{4}, J_{3}$, and $J_{4}$ (see Table 1).
} 
(A.4), and (A.5) and linearizing we obtain

$\frac{\mathrm{d}}{\mathrm{d} \tau} \delta y=-\left(a_{1}+a_{2}\right) \delta y+a_{3} y_{\infty} \delta z$,

$\frac{\mathrm{d}}{\mathrm{d} \tau} \delta z=-c_{2} \frac{P}{B+P} \delta z$

$\frac{\mathrm{d}}{\mathrm{d} \tau} \delta u=-\left(d_{2}+d_{1} y_{\infty}\right) \delta u-d_{1} u_{\infty} \delta y$.

The corresponding eigenvalues satisfy the following characteristic equation:

$$
\begin{aligned}
& \left(a_{1}+a_{2}+a_{3} z_{\infty}+\lambda\right)\left(d_{2}+d_{1} y_{\infty}+\lambda\right) \\
& \quad \times\left(c_{2} \frac{P}{B+P}+\lambda\right)=0 .
\end{aligned}
$$

While all roots of Eq. (A.12) are real and negative, $m_{\infty}=0$ is an unstable fixed point of Eq. (A.3). Thus the critical point is unstable, so that the cell will grow and start its progression through the cell-cycle. However, since Eq. (A.3) decouples and can be integrated, the mass can be considered as a forcing term for the rest of the system. If we follow Tyson and Novak (2001) and consider $m$ in Eq. (A.2) as a control parameter which varies in time, then the dynamical system Eqs. (A.13)(A.17) has a stable branch for values of $m>0$ and smaller than some critical value (see Fig. 12(a)).

In any case, what is important here is that a fixed point corresponding to the $\mathrm{G}_{1}$ phase of the cell-cycle $(x \simeq 1$ and $y \ll x)$ exists for $m=0$ but not for $m=m_{*}$.

The analysis for $x=\varepsilon \hat{x}$ is very similar. In this case we have that $1-x \gg J_{3}$ (see Table 1), so that, to leading order:

$\varepsilon \frac{\mathrm{d} \hat{x}}{\mathrm{~d} \tau}=\left(1+b_{3} u\right)-\frac{b_{4} m y \hat{x}}{\hat{J}_{4}+\hat{x}}$,

$\frac{\mathrm{d} y}{\mathrm{~d} \tau}=a_{4}-\left(a_{1}+a_{3} z\right) y$,

$\frac{\mathrm{d} m}{\mathrm{~d} \tau}=\eta m\left(1-\frac{m}{m_{*}}\right)$,

$\frac{\mathrm{d} z}{\mathrm{~d} \tau}=c_{1}\left(1-\frac{m}{m_{*}}\right)-c_{2} \frac{P}{B+P} z$,

$\frac{\mathrm{d} u}{\mathrm{~d} \tau}=d_{2}(1-u)-d_{1} y u$,

where $\hat{J}_{4}=\varepsilon^{-1} J_{4}=O(1)$. As above Eq. (A.15), which decouples, has two fixed points $m_{\infty}=0$ and $m_{\infty}=m_{*}$. If $m_{\infty}=0$, then Eq. (A.13) yields a contradiction: $u_{\infty}<0$. So we conclude that a fixed point to Eqs. (A.13)-(A.17) with $m_{\infty}=0$ is not physically realistic. When $m_{\infty}=m_{*}$, Eq. (A.16) yields $z_{\infty}=0$ while Eqs. (A.14) and (A.17) imply

$y_{\infty}=\frac{a_{4}}{a_{1}}$, $u_{\infty}=\frac{d_{2}}{d_{2}+d_{1} y_{\infty}}$

Hence to leading order $O\left(\varepsilon^{0}\right)$ the physically realistic fixed point of Eqs. (A.13)-(A.17) has $x_{\infty}=0, m_{\infty}=m_{*}$, $z_{\infty}=0$, with $y_{\infty}$ and $u_{\infty}$ given by Eqs. (A.18) and (A.19).

The linear stability of this fixed point is determined as in the previous case. Linearizing Eqs. (A.14), (A.15), and (A.17) we obtain the following characteristic equation:

$\left(a_{1}+\lambda\right)\left(d_{2}+d_{1} y_{\infty}+\lambda\right)\left(c_{2} \frac{P}{B+P}+\lambda\right)=0$.

Since all the roots of this equation are real and negative, and Eq. (A.16) decouples with $m_{\infty}=m_{*}$ a stable fixed point, this fixed point is a linearly stable steady state of Eqs. (A.13)-(A.17).

The analysis of the first order correction, $\hat{x}$, to the trivial steady state clarifies the main difference between the normal and cancer cell-cycle models, i.e. the ability to produce quiescence. Using Eq. (A.13) it is possible to show that the first-order correction to $x_{\infty}$ is given by

$\hat{x}_{\infty}=\frac{\hat{J}_{4}}{\left(\left(b_{4} m_{*} y_{\infty}\right) /\left(1+b_{3} u_{\infty}\right)\right)-1}$,

which for physically realistic solutions yields the condition:

$\frac{b_{4} m_{*} y_{\infty}}{1+b_{3} u_{\infty}}>1$.

It is easy to check that this condition is satisfied if $y_{\infty}>$ $\bar{y}_{\infty}$ where $\bar{y}_{\infty}$ is given by (see Eq. (A.19)):

$\bar{y}_{\infty}=\frac{A_{1}+\sqrt{A_{1}^{2}+4 A_{0} a_{2}}}{2 A_{2}}$,

with $A_{0}=d_{2}+b_{3} d_{1}, \quad A_{1}=d_{1}-b_{4} d_{2} m_{*}, \quad$ and $A_{2}=$ $b_{4} d_{1} m_{*}$. Eqs. (A.18) and (A.23) yield a condition on the model parameters in order to obtain a consistent (positive) value of $\hat{x}$. Provided this condition is satisfied, we obtain a feasible value of $x_{\infty}$ regardless of the extracellular oxygen tension, $P$. Physically this means that towards the end of the cell-cycle the cell is automatically in the $\mathrm{S}-\mathrm{G}_{2}-\mathrm{M}$ phase and thus the normal model cannot produce quiescence. As we show in Section 4.3 the situation for our cancer cell-cycle model is different.

\section{A.2. Fixed points and linear stability analysis of the cancer cell model}

Following the same basic procedure outlined in Section 4.2 , the linear stability of the cancer model is now analysed by focusing on the physically relevant situations for which $x \simeq 0$ or $x \simeq 1$. 
When $x=1-\varepsilon \tilde{x}$, the model equations simplify to give

$\varepsilon \frac{\mathrm{d} \tilde{x}}{\mathrm{~d} \tau}=b_{4} m y-\varepsilon\left(1+b_{3} u\right) \tilde{x}$,

$\frac{\mathrm{d} y}{\mathrm{~d} \tau}=a_{4}-\left(a_{1}+a_{2}+a_{3} z\right) y$,

$\frac{\mathrm{d} m}{\mathrm{~d} \tau}=\eta m\left(1-\frac{m}{m_{*}}\right)$,

$\frac{\mathrm{d} z}{\mathrm{~d} \tau}=c_{1}-c_{2} \frac{P}{B+P} z$

$\frac{\mathrm{d} u}{\mathrm{~d} \tau}=d_{2}(1-u)-d_{1} y u$.

If $m_{\infty}=0$, we obtain

$z_{\infty}=\frac{c_{1}(B+P)}{c_{2} P}$

$y_{\infty}=\frac{a_{4}}{a_{1}+a_{2}+a_{3} z_{\infty}}$,

$u_{\infty}=\frac{d_{2}}{d_{2}+d_{1} y_{\infty}}$

and $x_{\infty}=1$ to leading order. The corresponding linear analysis (in which only Eqs. (A.25), (A.27) and (A.28) are considered) leads to the following characteristic equation:

$$
\begin{aligned}
& \left(a_{1}+a_{2}+a_{3} z_{\infty}+\lambda\right)\left(d_{2}+d_{1} y_{\infty}+\lambda\right) \\
& \quad \times\left(c_{2} \frac{P}{B+P}+\lambda\right)=0,
\end{aligned}
$$

all of whose roots are real and negative. As for the normal cell model, $m_{\infty}=0$ is an unstable fixed point of Eq. (A.3) which decouples. Viewing $m$ as a timedependent control parameter, we say that the dynamical system possesses a stable branch for values of $m$ smaller than some critical value (see Fig. 12(c)).

As before, $m_{\infty}=m_{*}$ is in general incompatible with Eq. (A.24), since the steady-state condition would read: $b_{4} m_{*} y_{\infty}=\varepsilon\left(1+b_{3} u_{\infty}\right) \hat{x}_{\infty}$. However, Eqs. (A.29) and (A.30) imply that if $P \rightarrow 0$ then $y_{\infty} \rightarrow 0$. Thus, there exists $P_{0}>0$ such that if $P<P_{0}$ then the term $b_{4} m_{*} y_{\infty} \simeq O(\varepsilon)$. This condition is satisfied when $z_{\infty} \simeq O\left(\varepsilon^{-1}\right)$, which yields $y_{\infty} \simeq O\left(\varepsilon^{2}\right)$. Therefore, for $P<P_{0}$, we make the following rescaling: $\hat{m}=m / m_{*}, \hat{z}=z / \varepsilon,{ }^{8}$ and $b_{4} m_{*}=$ $\varepsilon^{-1} \hat{b}_{4}$, so that we can rewrite Eqs. (A.24)-(A.28) as

$\varepsilon \frac{\mathrm{d} \hat{x}}{\mathrm{~d} \tau}=\varepsilon^{-1} \hat{b}_{4} \hat{m} y-\varepsilon\left(1+b_{3} u\right) \hat{x}$,

$\frac{\mathrm{d} y}{\mathrm{~d} \tau}=a_{4}-\varepsilon^{-1} a_{3} \hat{z} y$,

\footnotetext{
${ }^{8} z$ becomes $O\left(\varepsilon^{-1}\right)$ only after some time, so that this scaling is only valid for $\tau \gg 1$.
}

$\frac{\mathrm{d} \hat{m}}{\mathrm{~d} \tau}=\eta \hat{m}(1-\hat{m})$

$\frac{\mathrm{d} \hat{z}}{\mathrm{~d} \tau}=\varepsilon c_{1}-c_{2} \frac{P}{B+P} \hat{z}$,

$\frac{\mathrm{d} u}{\mathrm{~d} \tau}=d_{2}(1-u)-d_{1} y u$.

Now, we can identify in Eq. (A.34) a small time scale, $t_{r}=\varepsilon / a_{3} \hat{z}$. Hence, we can apply the quasi-steady state approximation to Eq. (A.34) to obtain

$y \simeq \varepsilon^{2} \frac{\hat{a}_{4}}{a_{3} \hat{z}}$,

where $\hat{a}_{4}=a_{4} / \varepsilon$. Upon substitution of Eq. (A.38) into Eq. (A.33) we obtain

$\frac{\mathrm{d} \hat{x}}{\mathrm{~d} \tau}=\frac{\hat{b}_{4} \hat{a}_{4} \hat{m}}{a_{3} \hat{z}}-\left(1+b_{3} u\right) \hat{x}$,

$\frac{\mathrm{d} \hat{m}}{\mathrm{~d} \tau}=\eta \hat{m}(1-\hat{m})$,

$\frac{\mathrm{d} \hat{z}}{\mathrm{~d} \tau}=\varepsilon c_{1}-c_{2} \frac{P}{B+P} \hat{z}$,

$\frac{\mathrm{d} u}{\mathrm{~d} \tau}=d_{2}(1-u)$.

Notice that, since $P \simeq O(\varepsilon)$, the right-hand side of Eq. (A.41) yields a consistent expansion (recall that this scaling is only valid for long times $\tau \gg 1$ ).

The corresponding fixed points are:

$\hat{z}_{\infty}=\frac{\varepsilon c_{1}(B+P)}{c_{2} P}$

$y_{\infty}=\varepsilon^{2} \frac{\hat{a}_{4}}{a_{3} \hat{z}_{\infty}}$,

$\hat{x}_{\infty}=\frac{\hat{b}_{4} \hat{a}_{4}}{a_{3} \hat{z}_{\infty}\left(1+b_{3}\right)}$,

$u_{\infty}=1$

and $\hat{m}_{\infty}=1$. Of course, $\hat{m}=0$ is also a fixed point of Eq. (A.40), but we are now investigating the behaviour of a fully grown cell. We remark that $\hat{z}_{\infty} \simeq O(1)$ since $P<P_{0}$ and, more importantly, that a similar analysis cannot be carried out with the normal cell-cycle model, since in that case $z_{\infty}=0$ and therefore $y_{\infty} \simeq O(\varepsilon)$.

The local stability of the fixed point identified in Eqs. (A.43)-(A.46) is determined by linearizing Eqs. (A.39)-(A.42):

$$
\begin{aligned}
\frac{\mathrm{d}}{\mathrm{d} \tau} \delta \hat{x}= & -b_{3} u_{\infty} \delta \hat{x}+\frac{\hat{b}_{4} \hat{a}_{4}}{a_{3} \hat{z}_{\infty}} \delta \hat{m} \\
& -\frac{\hat{b}_{4} \hat{a}_{4} \hat{m}_{\infty}}{a_{3} \hat{z}_{\infty}^{2}} \delta \hat{z}-b_{3} \hat{x}_{\infty} \delta u,
\end{aligned}
$$

$\frac{\mathrm{d}}{\mathrm{d} \tau} \delta \hat{m}=-2 \eta \delta \hat{m}$ 
$\frac{\mathrm{d}}{\mathrm{d} \tau} \delta \hat{z}=-c_{2} \frac{P}{B+P} \delta \hat{z}$,

$\frac{\mathrm{d}}{\mathrm{d} \tau} \delta u=-d_{2} \delta u$,

where $\delta \hat{x}=\hat{x}-\hat{x}_{\infty}, \delta \hat{m}=\hat{m}-\hat{m}_{\infty}, \delta \hat{z}=\hat{z}-\hat{z}_{\infty}$, and $\delta u=u-u_{\infty}$. The corresponding characteristic equation for the eigenvalues $\lambda$ is

$\left(b_{3} u_{\infty}+\lambda\right)(2 \eta+\lambda)\left(d_{2}+\lambda\right)\left(c_{2} \frac{P}{B+P}+\lambda\right)=0$,

which yields negative eigenvalues. We have performed simulations to check the asymptotics (results not shown). We have found that our analytical calculation is in excellent agreement with the numerics, but only for values of the oxygen concentration deep inside the hypoxic regime $\left(P \simeq 10^{-4}\right)$. However, the asymptotics fails to predict the critical value of the oxygen concentration for hypoxia-induced quiescence ( $P \simeq 0.008$, see Fig. 6). This is due to the fact that, for the cancer cell-cycle model, the validity of the approximation $x=1-\varepsilon \tilde{x}$ depends on the oxygen level: it is a good approximation for very low levels of oxygen.

A similar analysis, carried out when $x=\varepsilon \hat{x}$, which corresponds to the end of the cell-cycle, yields

$\varepsilon \frac{\mathrm{d} \hat{x}}{\mathrm{~d} \tau}=\left(1+b_{3} u\right)-\frac{b_{4} m y \hat{x}}{\hat{J}_{4}+\hat{x}}$,

$\frac{\mathrm{d} y}{\mathrm{~d} \tau}=a_{4}-\left(a_{1}+a_{3} z\right) y$,

$\frac{\mathrm{d} m}{\mathrm{~d} \tau}=\eta m\left(1-\frac{m}{m_{*}}\right)$,

$\frac{\mathrm{d} z}{\mathrm{~d} \tau}=c_{1}-c_{2} \frac{P}{B+P} z$,

$\frac{\mathrm{d} u}{\mathrm{~d} \tau}=d_{2}(1-u)-d_{1} y u$.

As in Section 4.2, the only physically realistic fixed point of Eq. (A.54) is $m_{\infty}=m_{*}$ (since $\left.m_{\infty}=0 \Rightarrow u_{\infty}<0\right)$ in which case:

$z_{\infty}=\frac{c_{1}(B+P)}{c_{2} P}$

$y_{\infty}=\frac{a_{4}}{a_{1}+a_{3} z_{\infty}}$,

$u_{\infty}=\frac{d_{2}}{d_{2}+d_{1} y_{\infty}}$

and $x_{\infty}=0$ to leading order. Again, given that Eq. (A.54) decouples and that $m_{\infty}=m_{*}$ is a stable fixed point of Eq. (A.54), we can study the linear stability of the system to leading order by linearizing Eqs. (A.53), (A.55) and (A.56). This process eventually leads to the following characteristic equation for the eigenvalues $\lambda$ :

$$
\begin{aligned}
& \left(a_{1}+a_{3} z_{\infty}+\lambda\right)\left(d_{2}+d_{1} y_{\infty}+\lambda\right) \\
& \times\left(c_{2} \frac{P}{B+P}+\lambda\right)=0 .
\end{aligned}
$$

All the roots of Eq. (A.60) are real and negative. As in the case of the normal cell-cycle model, the first-order correction terms yield important information about the behaviour of the system. From Eq. (A.52) with $x=\varepsilon \hat{x}$ we have that

$\hat{x}_{\infty}=\frac{\hat{J}_{4}}{\left(b_{4} m_{*} y_{\infty} / 1+b_{3} u_{\infty}\right)-1}$,

which leads to the condition:

$\frac{b_{4} m_{*} y_{\infty}}{1+b_{3} u_{\infty}}>1$.

After some algebra, we deduce that this condition is satisfied if $y_{\infty}>\bar{y}_{\infty}$ where $\bar{y}_{\infty}$ is given by Eq. (A.23).

From Eqs. (A.57) and (A.58), we can see that the condition $y_{\infty}>\bar{y}_{\infty}$ implies the existence of a threshold oxygen concentration, $\bar{P}$, such that $\hat{x}_{\infty}<0$ if $P<\bar{P}$. Thus, if $P<\bar{P}$ there is no physically realistic fixed point of Eqs. (A.52)-(A.56). Biologically, this means that the control system of the cell-cycle gets stuck in the $G_{1}$ phase, thus yielding quiescent behaviour. On the contrary, for $P>\bar{P}$ a linearly stable (of type $\mathrm{S}-\mathrm{G}_{2}-\mathrm{M}$ ) fixed point exists.

We have shown that our cancer cell model has a fixed point with $m_{\infty}=0$ which corresponds to $\mathrm{G}_{1}$ (high values of $x=[\mathrm{Cdh}]$ and low values of $y=[\mathrm{Cyc}]$ ), whereas when $m_{\infty}=m_{*}$ a stable fixed point corresponding to $\mathrm{S}-\mathrm{G}_{2}-\mathrm{M}$ (low values of $x=[\mathrm{Cdh}]$ and high values of $y=[\mathrm{Cyc}]$ ) exists only if the oxygen level exceeds a critical value. When the oxygen tension is below this critical value, the cancer model has a $\mathrm{G}_{1}$-like fixed point which is stable when $m_{\infty}=m_{*}$, meaning that the cancer cell-cycle model does not go through the $\mathrm{G}_{1} / \mathrm{S}$ transition; this explains why the cancer cell-cycle model is able to produce quiescence.

\section{References}

Alberts, B., Bray, D., Lewis, J., Raff, M., Roberts, K., Watson, J.D., 1994. Molecular Biology of the Cell, 3rd Edition. Garland Publishing, New York, USA.

Bai, M., Vlachonikolis, J., Agnantis, N.J., Tsanou, E., Dimou, S., Nicolaides, C., Stefanaki, S., Pavlidis, N., Kanavarous, P., 2001. Low expression of $\mathrm{p} 27$ protein combined with altered $\mathrm{p} 53$ and $\mathrm{Rb} /$ p16 expression status is associated with increased expression of cyclin A and cyclin B1 in diffuse B-cell lymphoma. Mod. Path. 14, 1105-1113.

Carmeliet, P., Dor, Y., Herbert, J.M., Fukumura, D., Brusselsmans, K., Dewerchin, M., Neeman, M., Bono, F., Abramovitch, R., Maxwell, P., Koch, C.J., Ratcliffe, P., Moons, L., Jain, R.K., Collen, D., Keshert, E., 1998. Role of HIF- $\alpha$ in hypoxia-mediated 
apoptosis, cell proliferation and tumour angiogenesis. Nature 394, 485-490.

Cherry, J.L., Adler, F.R., 2000. How to make a biological switch. J. Theor. Biol. 203, 117-133.

DeGregori, J., Kowalik, T., Nevins, J.R., 1995. Cellular targets for activation by the E2F1 transcription factor include DNA synthesis and $\mathrm{G}_{1} / \mathrm{S}$-regulatory genes. Mol. Cell Biol. 15, 4215-4224.

Deutsch, A., Dormann, S., 2002. Modelling avascular tumour growth with a hybrid cellular automaton. In Silico Biol. 2, $1-14$.

Dhillon, N.K., Mudryj, M., 1995. Ectopic expression of cyclin E in estrogen responsive cell abrogates antiestrogeb mediated growth arrest. Oncogene 21, 4626-4634.

Folkman, J., Hochberg, M., 1973. Self-regulation of growth in three dimensions. J. Exp. Med. 138, 745-753.

Funk, J.O., 1999. Cancer cell-cycle control. Anticancer Res. 19, 4772-4780.

Gammack, D., Byrne, H.M., Lewis, C.E., 2001. Estimating the selective advantage of mutant $\mathrm{p} 53$ tumour cells to repeated rounds of hypoxia. Bull. Math. Biol. 63, 135-166.

Gardner, L.B., Li, Q., Parks, M.S., Flanagan, W.M., Semenza, G.L., Dang, C.V., 2001. Hypoxia inhibits $\mathrm{G}_{1} / \mathrm{S}$ transition through regulation of p27 expression. J. Biol. Chem. 276, 7919-7926.

Hamamoto, R., Yamada, K., Kamahira, M., Iijima, S., 1998. Differentiation and proliferation of primary rat hepatocytes cultured as spheroids. J. Biochem. 124, 972-979.

Katayama, N., Mahmud, N., Nishii, K., Onishi, K., Masuya, M., Mitani, H., Araki, H., Suzuki, H., Kobayashi, K., Minami, N., Shiku, H., 2000. Bcl-2 in cell-cycle regulation of hematopoietic cells by transforming growth factor-beta 1. Leukemia Lymphoma 39, 601-605.

Kinzler, K.W., Vogelstein, B., 1996. Life (and death) in a malignant tumour. Nature 379, 19-20.

Kirla, R.M., Haapasalo, H.K., Kalimo, H., Salminen, E.K., 2003. Low expression of p27 indicates a poor prognosis in patients with high garde astrocytomas. Cancer 97, 644-648.
Knudsen, K.E., Fribourg, A.F., Strobeck, M.W., Blanchard, J.M., Knudsen, E.S., 1999. Cyclin A is a functional target of retinoblastoma tumour suppressor protein-mediated cell-cycle arrest. J. Biol. Chem. 274, 27632-27641.

Leshem, Y., Halevy, O., 2002. Phosphorylation of $\mathrm{pRb}$ is required for HGF-induced muscle cell proliferation and is p27(kip1)-dependent. J. Cell Physiol. 191, 173-182.

Nasmyth, K., 1995. Evolution of the cell-cycle. Trans. Philos. Soc. Roy. Soc. B 349, 271-281.

Park, C., Lee, I., Kang, W.K., 2001. Lovastatin-induced E2F-1 modulation and its effect on prostate cancer cell death. Carcinogenesis 22, 1727-1731.

Philipp-Staheli, J., Payne, S.R., Kemp, C.J., 2001. p27(Kip1): regulation and function of haploinsufficient tumour supressor and its misregulation in cancer. Exp. Cell Res. 264, 148-168.

Press, W.H., Flannery, B.P., Teukolsky, S.A., Vetterling, W.T., 1992. Numerical Recipes in C. Cambridge University Press, Cambridge, UK.

Royds, J.A., Dower, S.K., Qwarnstrom, E.E., Lewis, C.E., 1998. Response of tumour cells to hypoxia: role of p53 and NFkB. J. Clin. Pathol. Mol. Pathol. 51, 55-61.

Saito, J., Khon, A.D., Roth, R.A., Noguchi, Y., Tatsumo, I., Hirai, A., Suzuki, K., Khon, L.D., Saji, M., Ringel, M.D., 2001. Regulation of FRTL-5 thyroid cell growth by phosphatidylinositol (OH) 3 kinase-dependent Akt-mediated signaling. Thyroid 11, 339-351.

Sauer, H., Ritgen, J., Heschler, J., Wartenberg, M., 1998. Hypotonic $\mathrm{Ca}^{2+}$ signaling and volume regulation in proliferating and quiescent cells from multicellular spheroids. J. Cell. Physiol. 175, 129-140.

Thomson, K.E., Royds, J.A., 1999. Hypoxia and reoxygenation: a pressure for mutant $\mathrm{p} 53$ cell selection and tumour progression. Bull. Math. Biol. 61, 759-778.

Tyson, J.J., Novak, B., 2001. Regulation of the eukariotic cell-cycle: molecular antagonism, hysteresis, and irreversible transitions. J. Theor. Biol. 210, 249-263. 